Network Working Group

Request for Comments: 3872

Category: Standards Track
D. Zinman

D. Walker

J. Jiang

September 2004

\title{
Management Information Base \\ for Telephony Routing over IP (TRIP)
}

Status of this Memo

This document specifies an Internet standards track protocol for the Internet community, and requests discussion and suggestions for improvements. Please refer to the current edition of the "Internet Official Protocol Standards" (STD 1) for the standardization state and status of this protocol. Distribution of this memo is unlimited.

Copyright Notice

Copyright (C) The Internet Society (2004).

Abstract

This memo defines a portion of the Management Information Base (MIB) module for use with network management protocols in the Internet community. In particular, it describes a set of managed objects that are used to manage Telephony Routing over IP (TRIP) devices.

Table of Contents

1. The Internet-Standard Management Framework . . . . . . . . . . 2

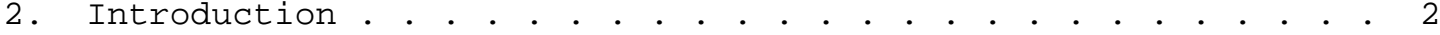

3. Conventions used in this document. . . . . . . . . . . . . . 2

4. Overview . . . . . . . . . . . . . . . . . . . . . . . . 2

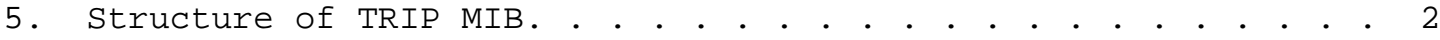

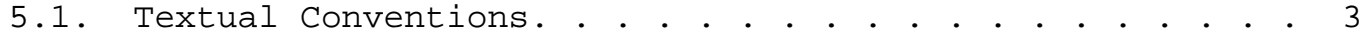

6. Definitions. . . . . . . . . . . . . . . . . . . . . . . 4

6.1. TRIP Textual Conventions . . . . . . . . . . . . . . . . 4

6.2. TRIP MIB . . . . . . . . . . . . . . . . . . . . . 7

7. Security Considerations. . . • . • . . . . . . . . . . . 48

8. References . . . . . . . . . . . . . . . . . . . . 50

8.1. Normative References . . . . . . . . . . . . . . 50

8.2. Informative References . . . . . . . . . . . . . . 51

9. Acknowledgments. . . . . . . . . . . . . . . . . . . 51

10. Authors' Addresses . . . . . . . . . . . . . . . . . . 52

11. Full Copyright Statement . . . . . . . . . . . . . 53 
1. The Internet-Standard Management Framework

For a detailed overview of the documents that describe the current Internet-Standard Management Framework, please refer to section 7 of RFC 3410 [RFC3410] .

Managed objects are accessed via a virtual information store, termed the Management Information Base or MIB. MIB module objects are generally accessed through the Simple Network Management Protocol (SNMP). Objects in this MIB module are defined using the mechanisms defined in the Structure of Management Information (SMI). This memo specifies a MIB module that is compliant to the SMIV2, which is described in STD 58, RFC 2578 [RFC2578], STD 58, RFC 2579 [RFC2579], and STD 58, RFC 2580 [RFC2580].

2. Introduction

This memo defines a portion of the Management Information Base (MIB) for use with network management protocols in the Internet community. In particular, it describes a set of managed objects that are used to schedule management operations periodically or at specified dates and times. Since TRIP [RFC3219] is modeled after the Border Gateway Protocol (BGP-4) [RFC1771], the managed objects for TRIP are also modeled after RFC1657 - Definitions of Managed Objects for the Fourth Version of the Border Gateway Protocol (BGP-4) using SMIv2 [RFC1657].

3. Conventions used in this document

The key words "MUST", "MUST NOT", "REQUIRED", "SHALL", "SHALL NOT", "SHOULD", "SHOULD NOT", "RECOMMENDED", "MAY", and "OPTIONAL" in this document are to be interpreted as described in BCP 14, RFC 2119 [RFC2119].

4. Overview

This MIB module provides managed objects for TRIP devices defined in Telephony Routing over IP [RFC3219]. TRIP is an inter-domain application-layer control protocol that exchanges information between TRIP location servers (LS) to provide efficient IP telephony routing.

\section{Structure of TRIP MIB}

This MIB module utilizes the framework described in RFC 2788 [RFC2788] for management of multiple instances of TRIP from a single entity. The Network Services Monitoring MIB module applTable will be populated with entries corresponding to each TRIP Location server 
in the system. Each TRIP Location Server will then have an applIndex associated with it. The value assigned to applIndex will represent the distinct instance of TRIP.

The TRIP MIB module contains the following groups of objects with each group as part of the management of a singular TRIP entity. Each group covers a section of functionality of TRIP:

- The tripconfigGroup contains the common configuration objects applicable to all TRIP applications referenced by the applindex.

- The trippeerTableconfigGroup contains the configuration objects applicable to all TRIP peers of the Location Server referenced by the applindex.

- The tripRouteGroup contains the configuration objects related to the routes of all TRIBs of this Location Server.

- The tripItadTopologyGroup contains information about the topology of the TRIP ITADs concerning this Location Server.

- The trippeerTablestatsGroup contains the statistical objects applicable to all TRIP peers of the Location Server referenced by the applindex.

- The tripNotificationGroup contains notifications that the TRIP application can generate.

- The tripNotifobjectGroup contains the objects needed by one or more of the notifications.

5.1. Textual Conventions

The data types TripItad and TripId are used as textual conventions in this document. A TRIP ITAD (IP Telephony Administrative Domain) is described in [RFC3219]. A TRIP ID is used as a distinct identifier for a TRIP Location Server. A TripAppProtocol is used to identify an application protocol. A TripAddressfamily is used to define an address family. Tripcommunityld is used as a distinct identifier for a TRIP community. TripProtocolversion depicts the version number of the TRIP protocol. TripsendReceiveMode describes the operational mode of the TRIP application. 
6. Definitions

6.1. TRIP Textual Conventions

TRIP-TC-MIB DEFINITIONS ::= BEGIN

IMPORTS

MODULE-IDENTITY,

Unsigned32,

Integer32,

mib-2

FROM SNMPV2-SMI

$--\quad[R F C 2578]$

TEXTUAL-CONVENTION

FROM SNMPV2-TC;

-- [RFC2579]

triPTC MODULE-IDENTITY

LAST-UPDATED "200409020000Z" -- Sep 02, 2004

ORGANIZATION "IETF IPTel Working Group.

Mailing list: iptelelists.bell-labs.com"

CONTACT-INFO

"Co-editor David Zinman

postal: 265 Ridley Blvd.

Toronto ON, M5M 4N8

Canada

email: dzinmanerogers.com

phone: +14164334298

Co-editor: David Walker

Sedna Wireless Inc.

postal: 495 March Road, Suite 500

ottawa, ON K2K $3 \mathrm{G} 1$

Canada

email: david.walker@sedna-wireless.com

phone: $\quad+16138788142$

Co-editor Jianping Jiang

Syndesis Limited

postal: $\quad 30$ Fulton Way

Richmond Hill, ON L4B 1 J5

Canada

email: jjiangesyndesis.com

phone: $\quad+1905886-7818 \times 2515$

DESCRIPTION

"Initial version of TRIP (Telephony Routing Over IP)

MIB Textual Conventions module used by other 


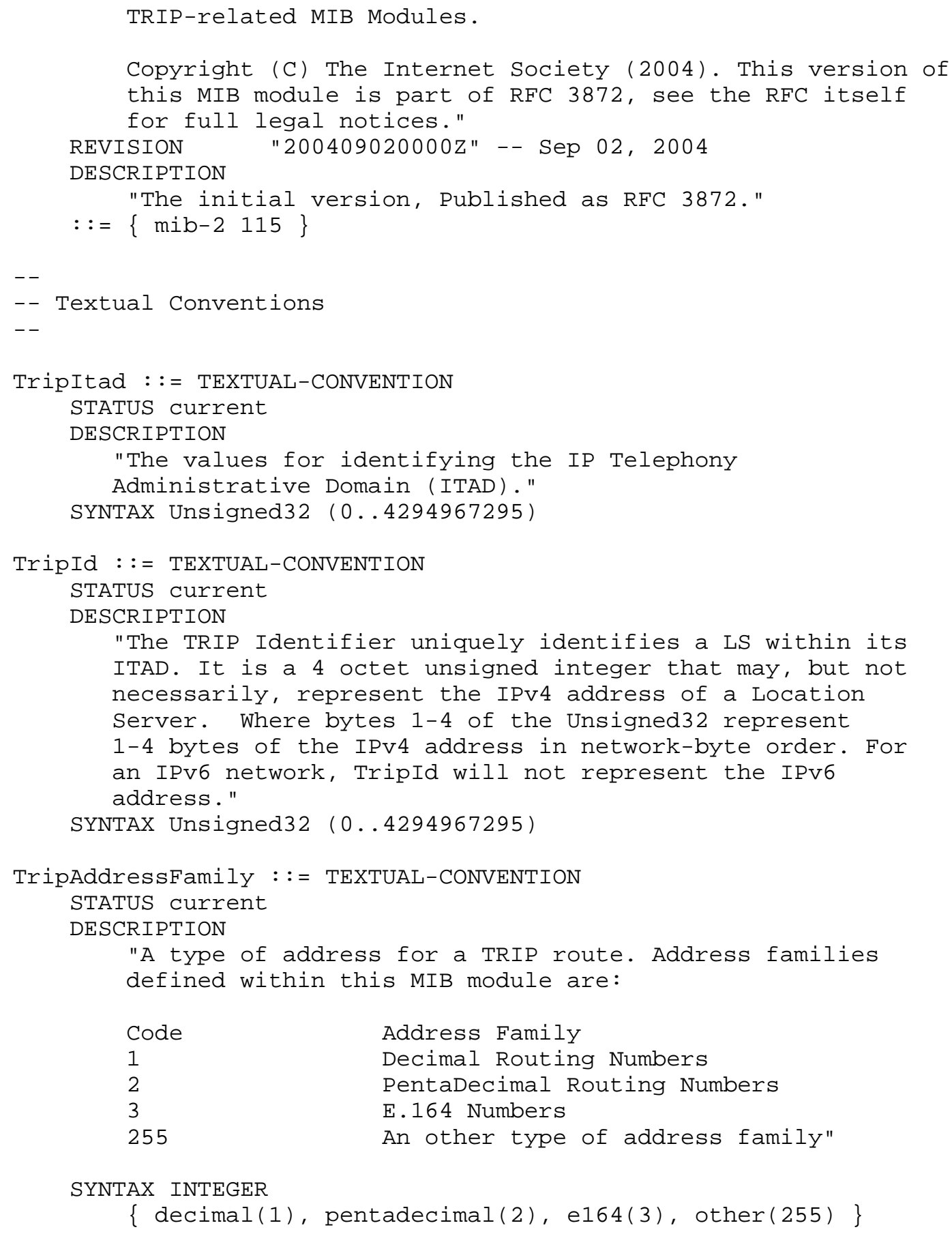




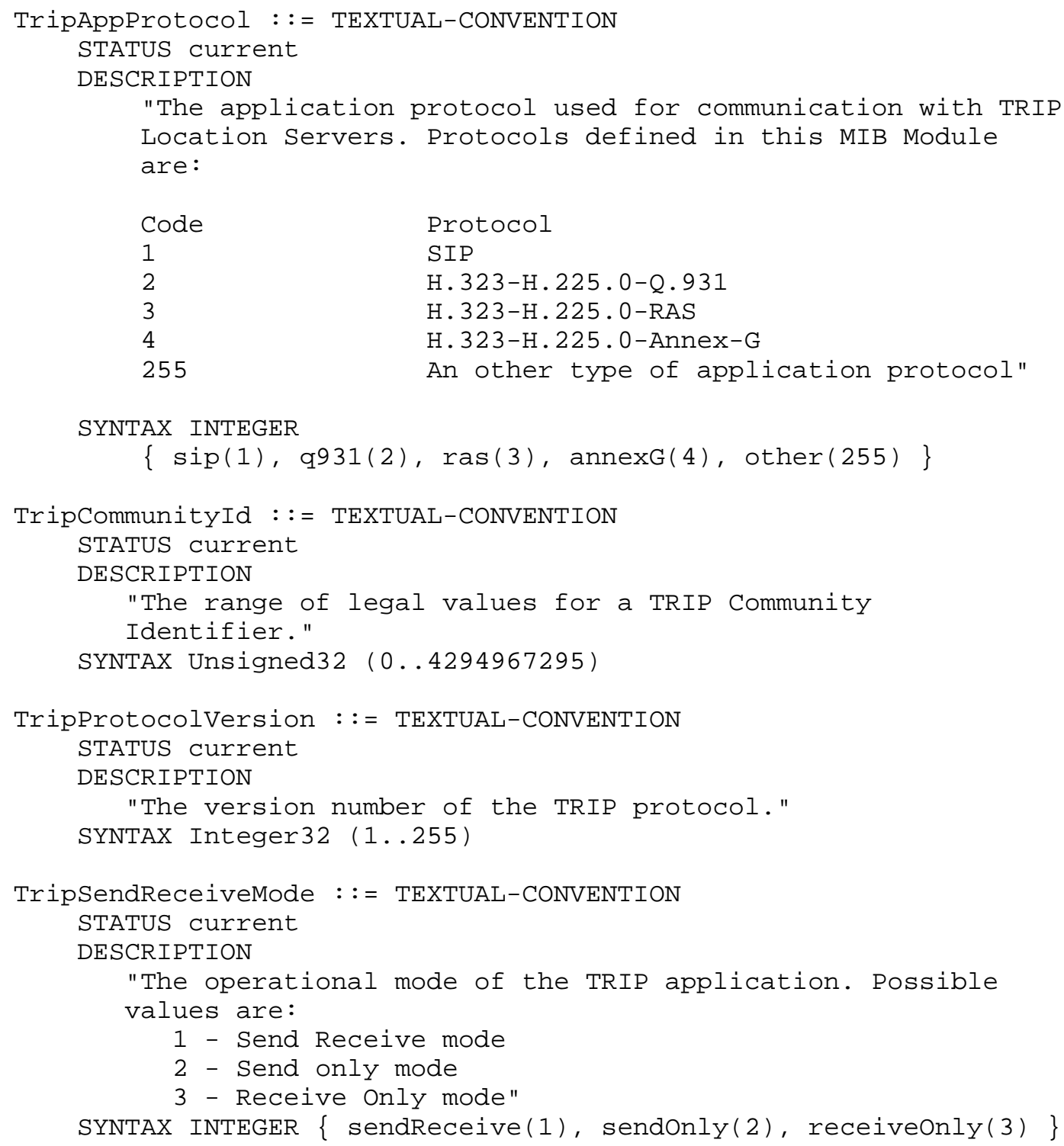




\subsection{TRIP MIB}

TRIP-MIB DEFINITIONS : := BEGIN

IMPORTS

MODULE-IDENTITY,

OBJECT-TYPE,

NOTIFICATION-TYPE,

Unsigned32,

Integer 32 ,

Counter32,

mib-2

FROM SNMPV2-SMI

$--\quad[R F C 2578]$

DateAndTime,

TimeInterval,

TruthValue,

Timestamp,

StorageType,

Rowstatus

FROM SNMPV2-TC

$--\quad[R F C 2579]$

OBJECT-GROUP, MODULE-COMPLIANCE, NOTIFICATION-GROUP

FROM SNMPV2-CONF

$--[R F C 2580]$

InetAddress Type,

InetAddress,

InetPort Number

FROM INET-ADDRESS-MIB -- [RFC3291]

appl Index, applRFC2788Group

FROM NETWORK-SERVICES-MIB -- [RFC2788]

TripItad,

TripId,

TripAppProtocol,

TripAddressfamily,

TripcommunityId,

TripProtocolVersion,

TripsendReceiveMode

FROM TRIP-TC-MIB; -- [RFC3872]

tripMIB MODULE-IDENTITY

LAST-UPDATED "200409020000Z" -- Sep 02, 2004

ORGANIZATION "IETF IPTel Working Group. 


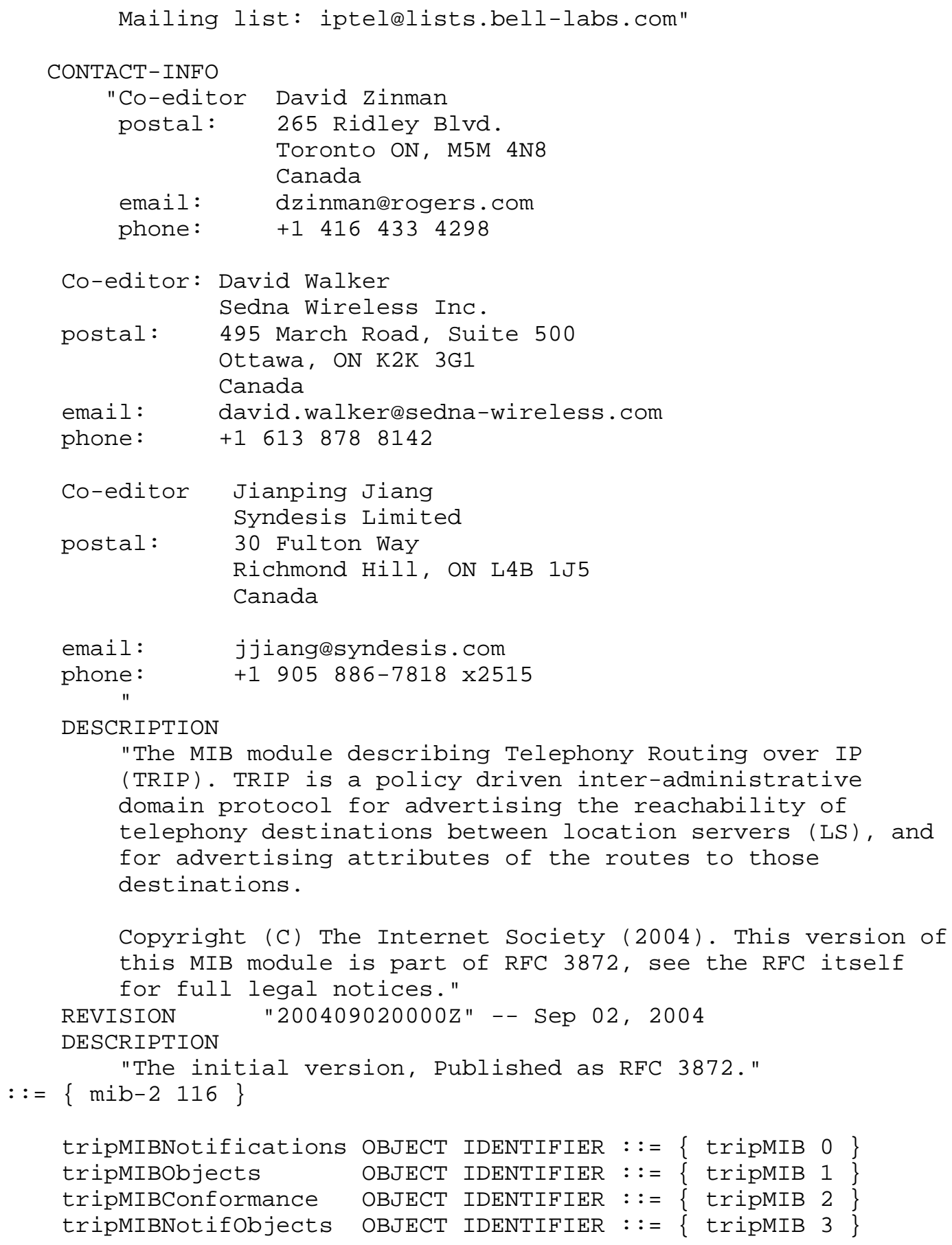




$\begin{array}{lcc}\text { tripMIBCompliances } & \text { OBJECT IDENTIFIER }::= \\ \text { tripMIBGroups } & \text { OBJECT IDENTIFIER }::= \\ & \{\text { tripMIBConformance } 2\}\end{array}$

$--$

- tripcfgTable

$--$

tripCfgTable OBJECT-TYPE

SYNTAX SEQUENCE OF TripCfgEntry

MAX-ACCESS not-accessible

STATUS current

DESCRIPTION

"This table contains the common configuration objects applicable to all TRIP applications referenced by the applindex. Each row represents those objects for a particular TRIP LS present in this system. The instances of TRIP LS's are uniquely identified by the applindex. The objects in this table sHOULD be nonvolatile and survive a reboot."

$::=\{$ tripMIBObjects 1$\}$

tripCfgEntry OBJECT-TYPE

SYNTAX TripCfgEntry

MAX-ACCESS not-accessible

STATUS current

DESCRIPTION

"A row of common configuration."

INDEX \{ applIndex $\}$

$::=\{$ tripcfigable 1$\}$

Tripcfgentry : : =

SEQUENCE \{

tripcfgProtocolversion TripprotocolVersion,

tripCfgItad

tripcfgIdentifier

TripItad,

tripcfgAdminstatus

TripId,

tripCfgoperstatus

INTEGER,

tripCfgAddrIAddrType

INTEGER,

InetAddress Type,

tripcfgAddr

InetAddress,

tripCfgPort

InetPortNumber,

tripCfgMinItadOriginationInterval

Unsigned32,

tripCfgMinRouteAdvertisementInterval Unsigned32,

tripCfgMaxPurgeTime

Unsigned32,

tripCfgDisableTime

Unsigned32,

tripCfgSendReceiveMode

TripsendReceiveMode,

tripcfgStorage

Storage Type

\} 


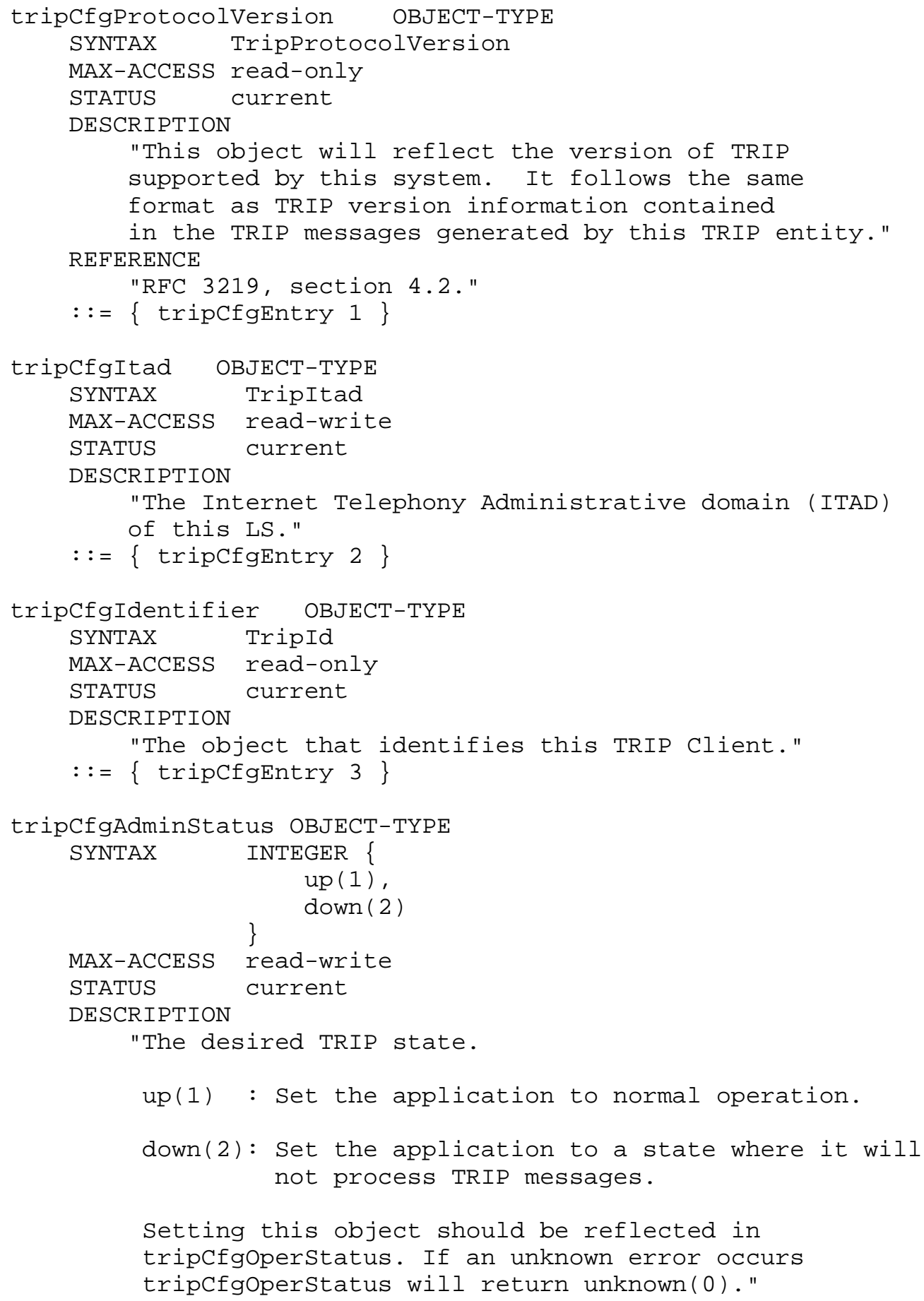




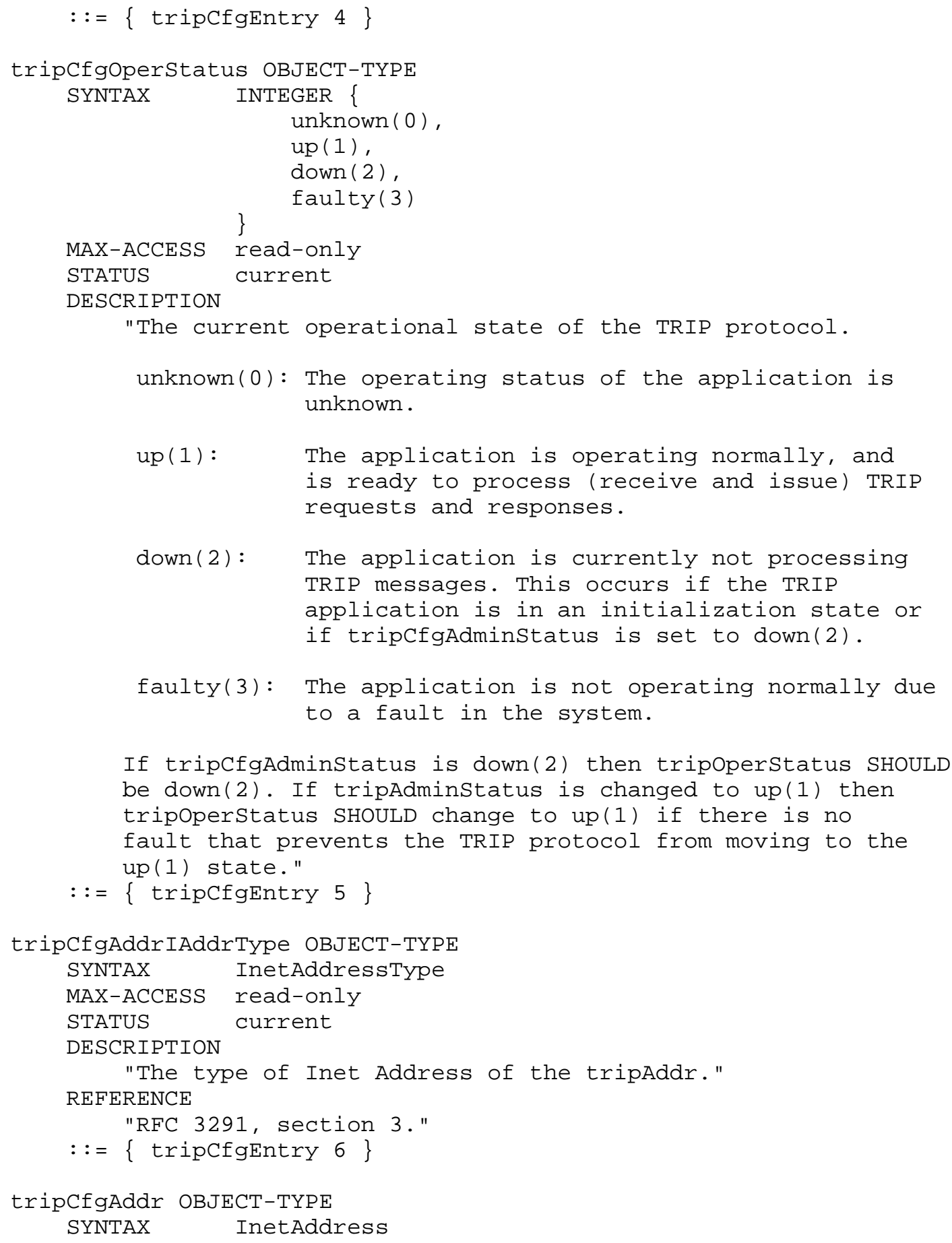




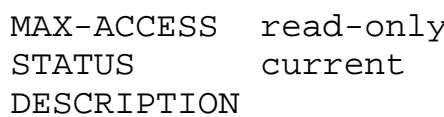

"The network address of the local LS that the peer connects to. The type of address depends on the object tripcfgAddrIAddrType. The type of this address is determined by the value of the tripcfgAddrIAddrType object." 


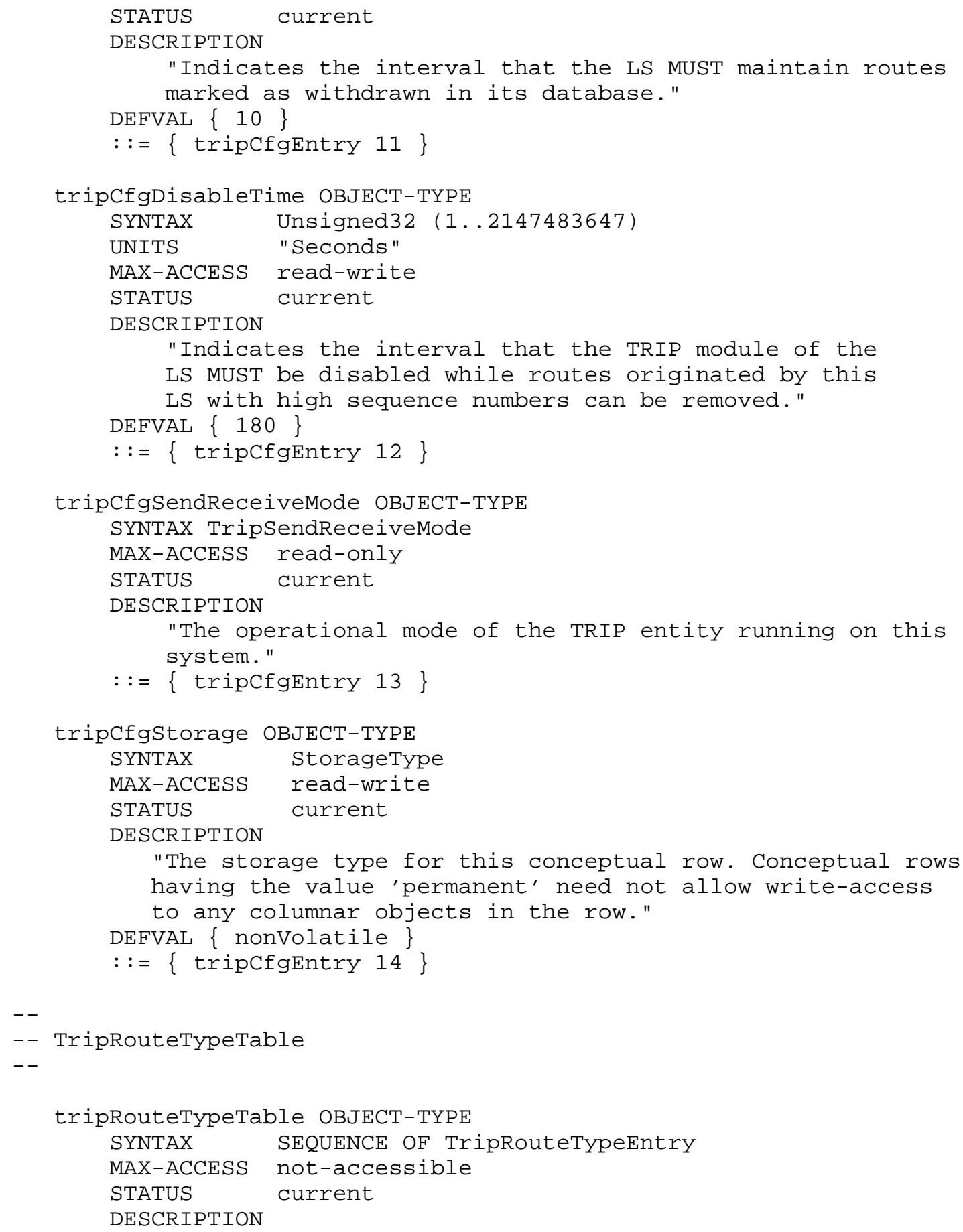




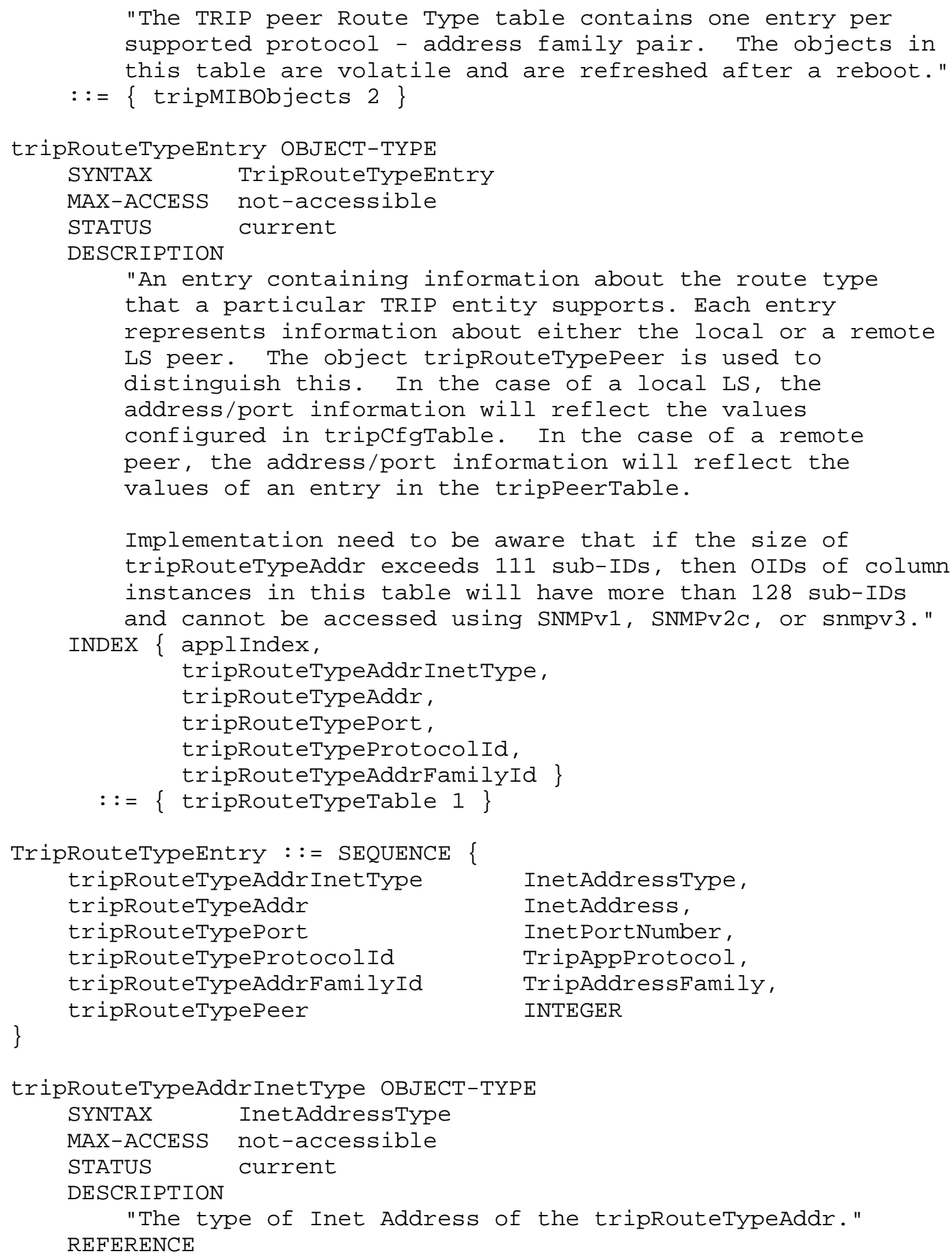




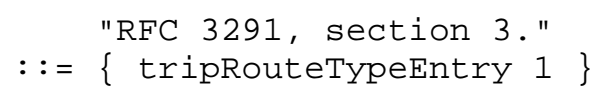




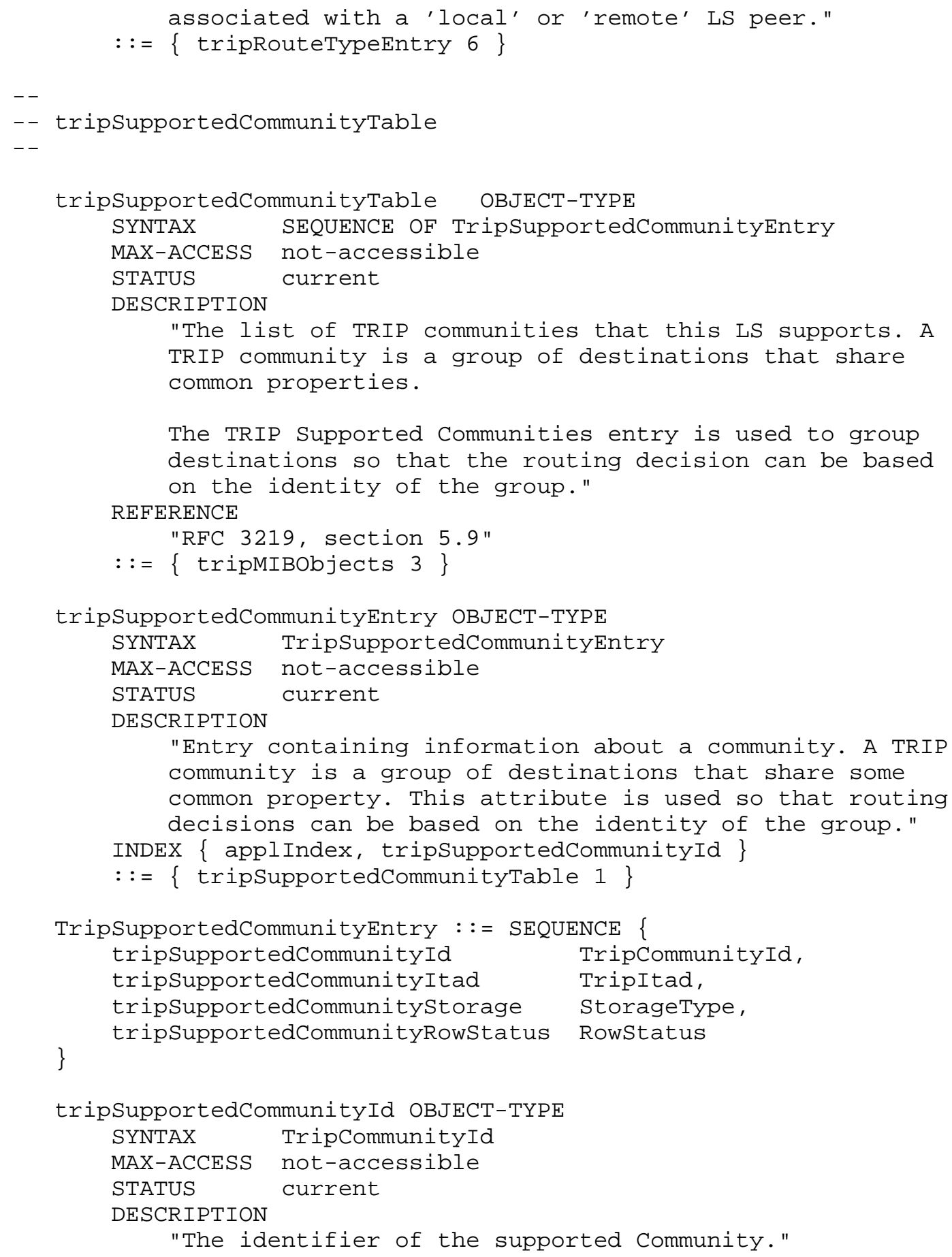




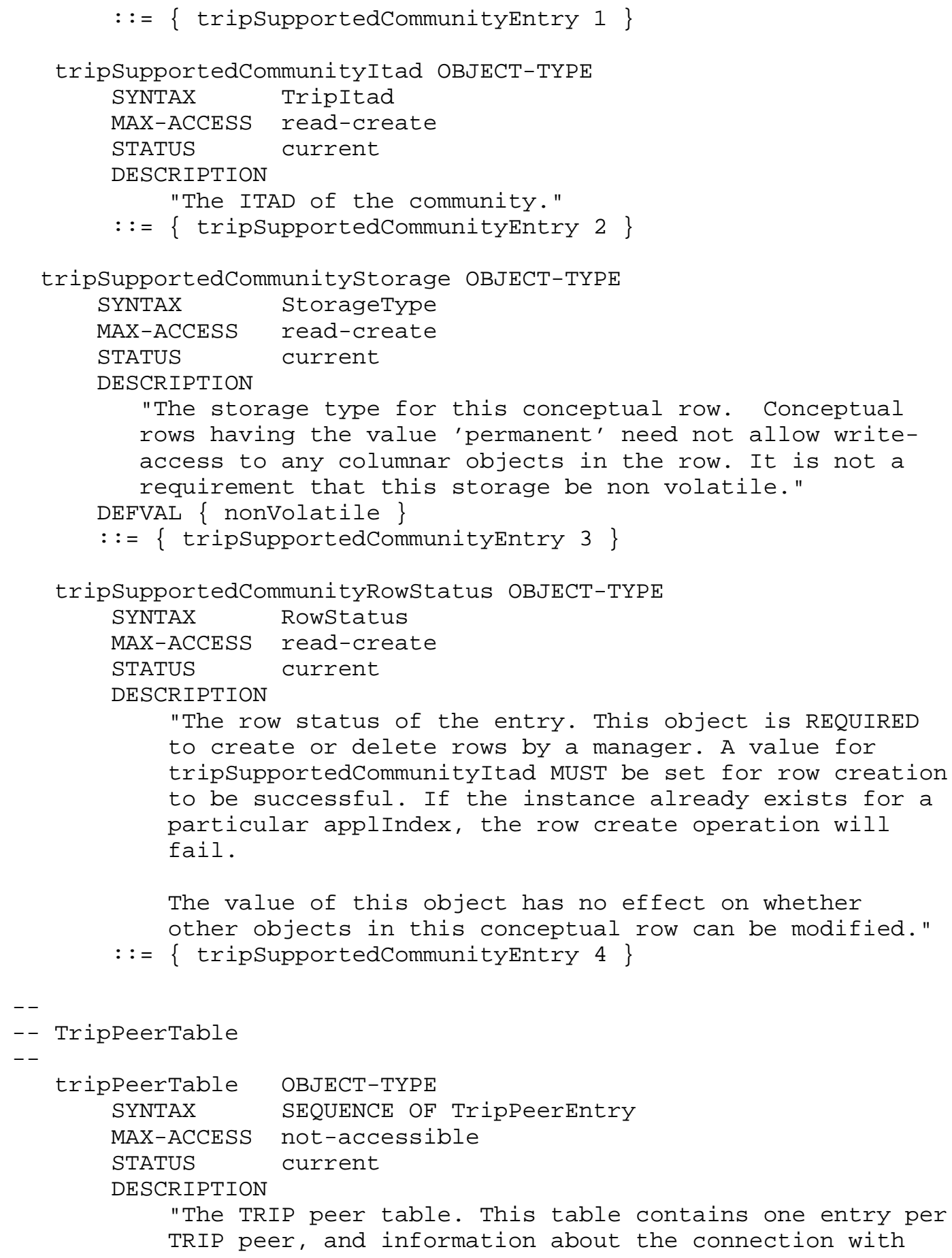




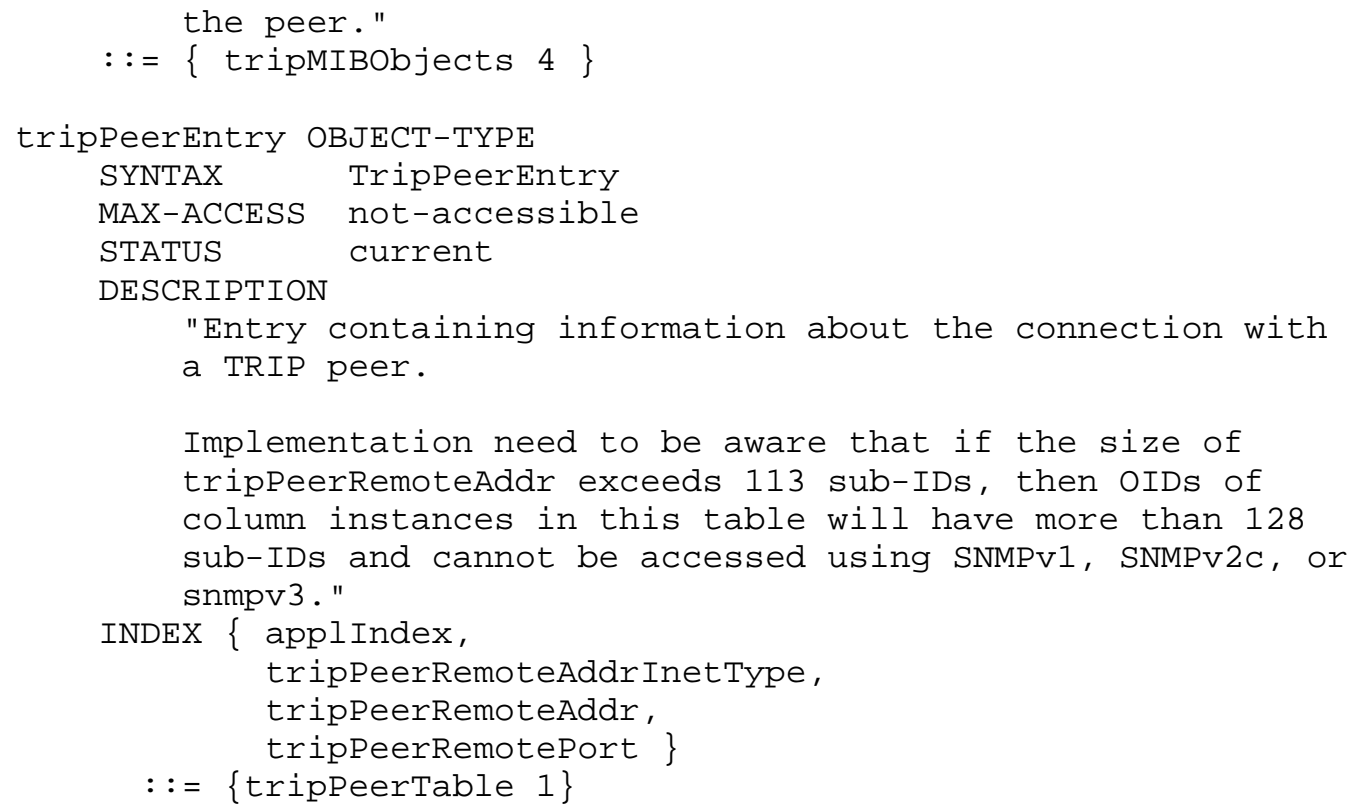

InetAddress Type, InetAddress, InetPortNumber, TripId, INTEGER, INTEGER, TripProtocolversion, TripSendReceiveMode, TripItad, Unsigned32, Unsigned32, Unsigned32, Unsigned32, Unsigned32, Unsigned32, Unsigned32, Unsigned32, TruthValue, StorageType, Rowstatus 


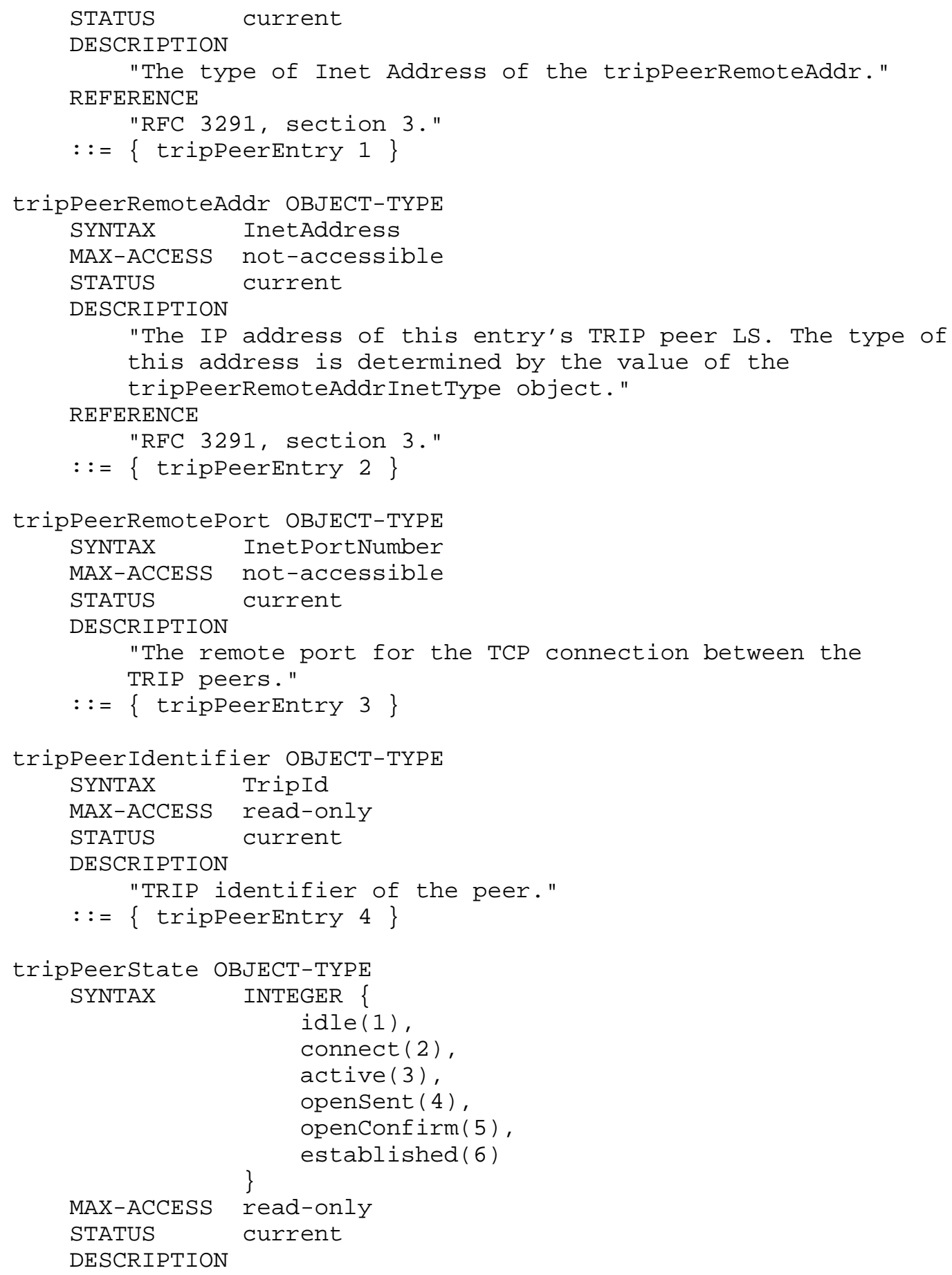


"TRIP Peer Finite state Machine state.

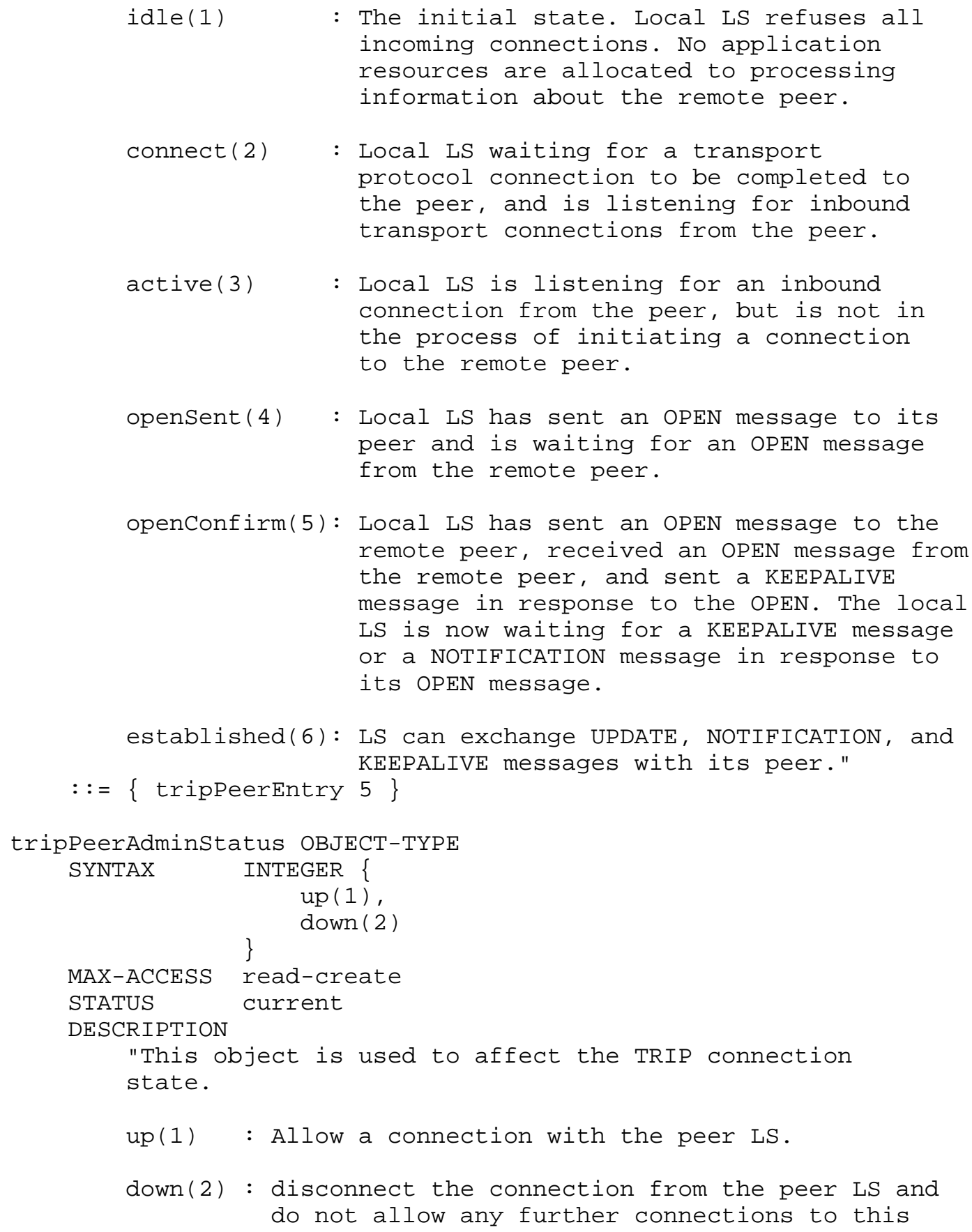




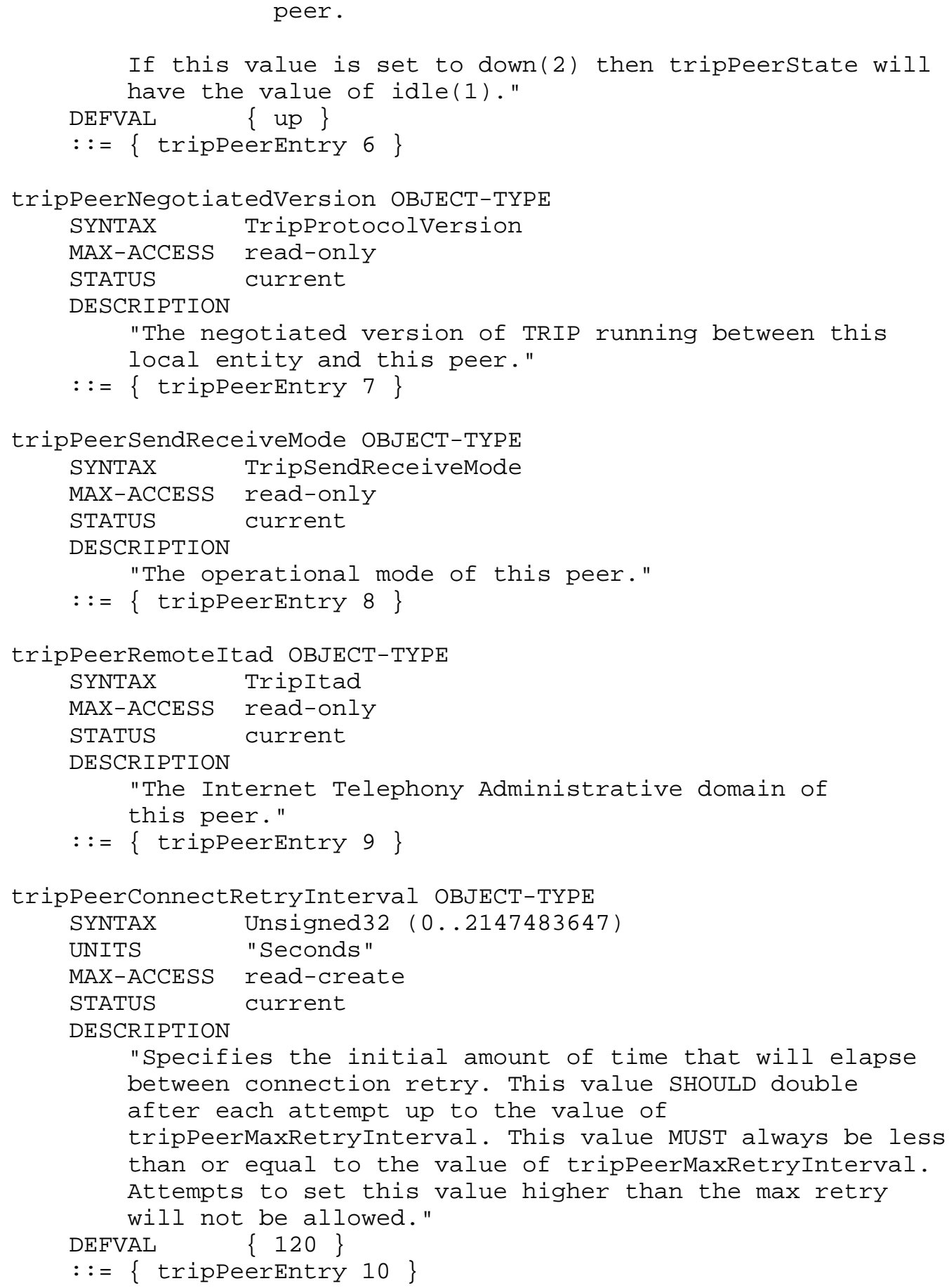




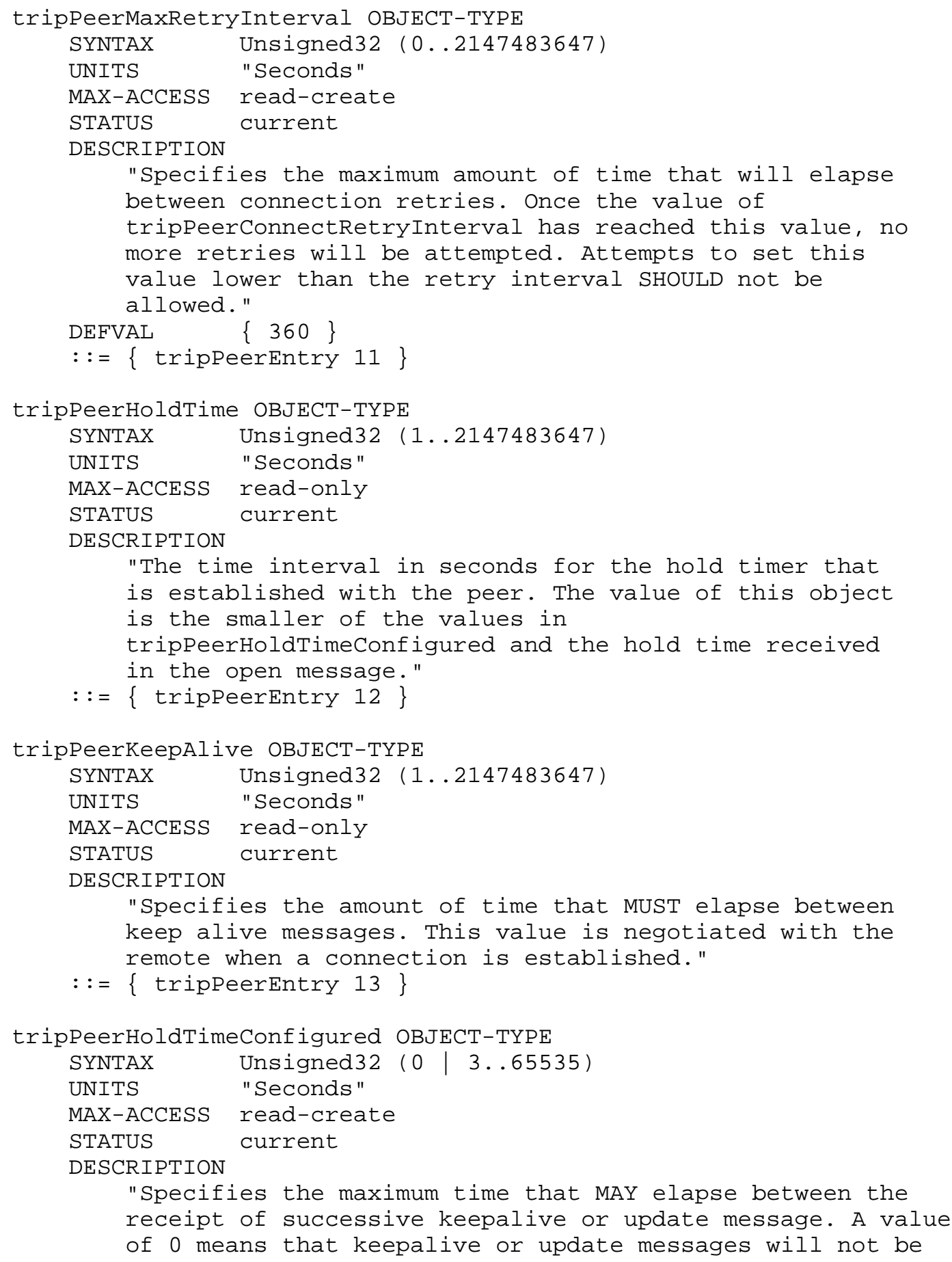




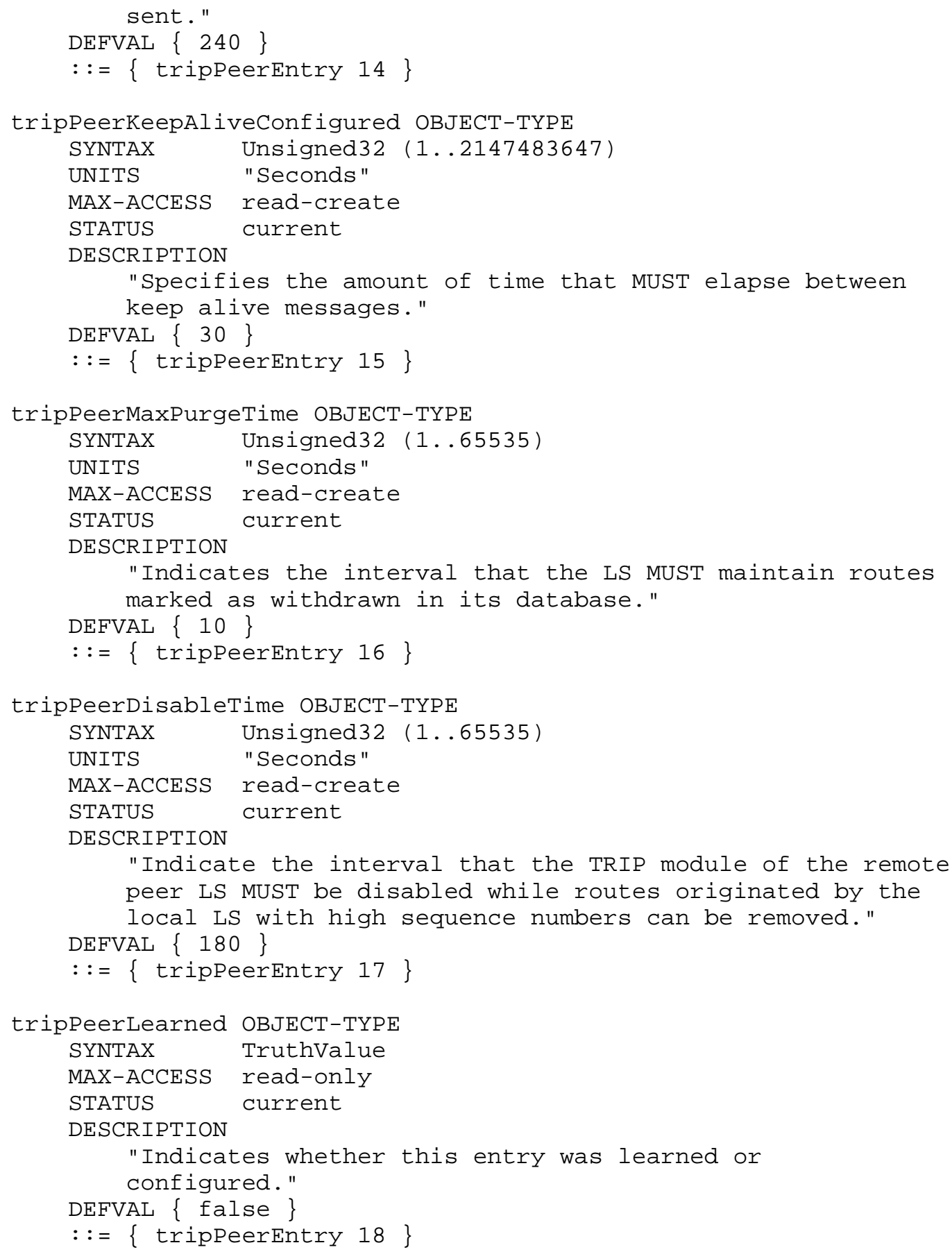




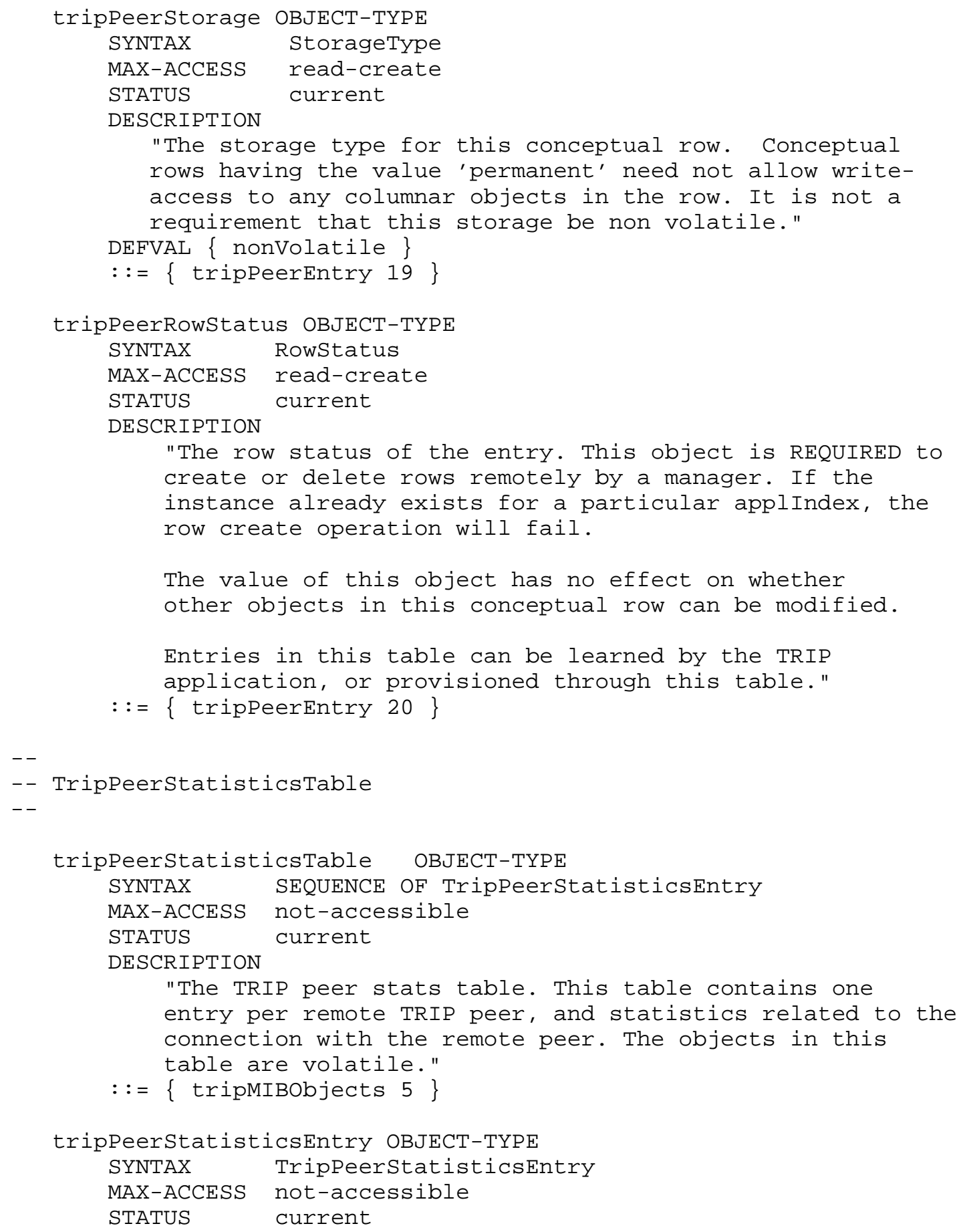




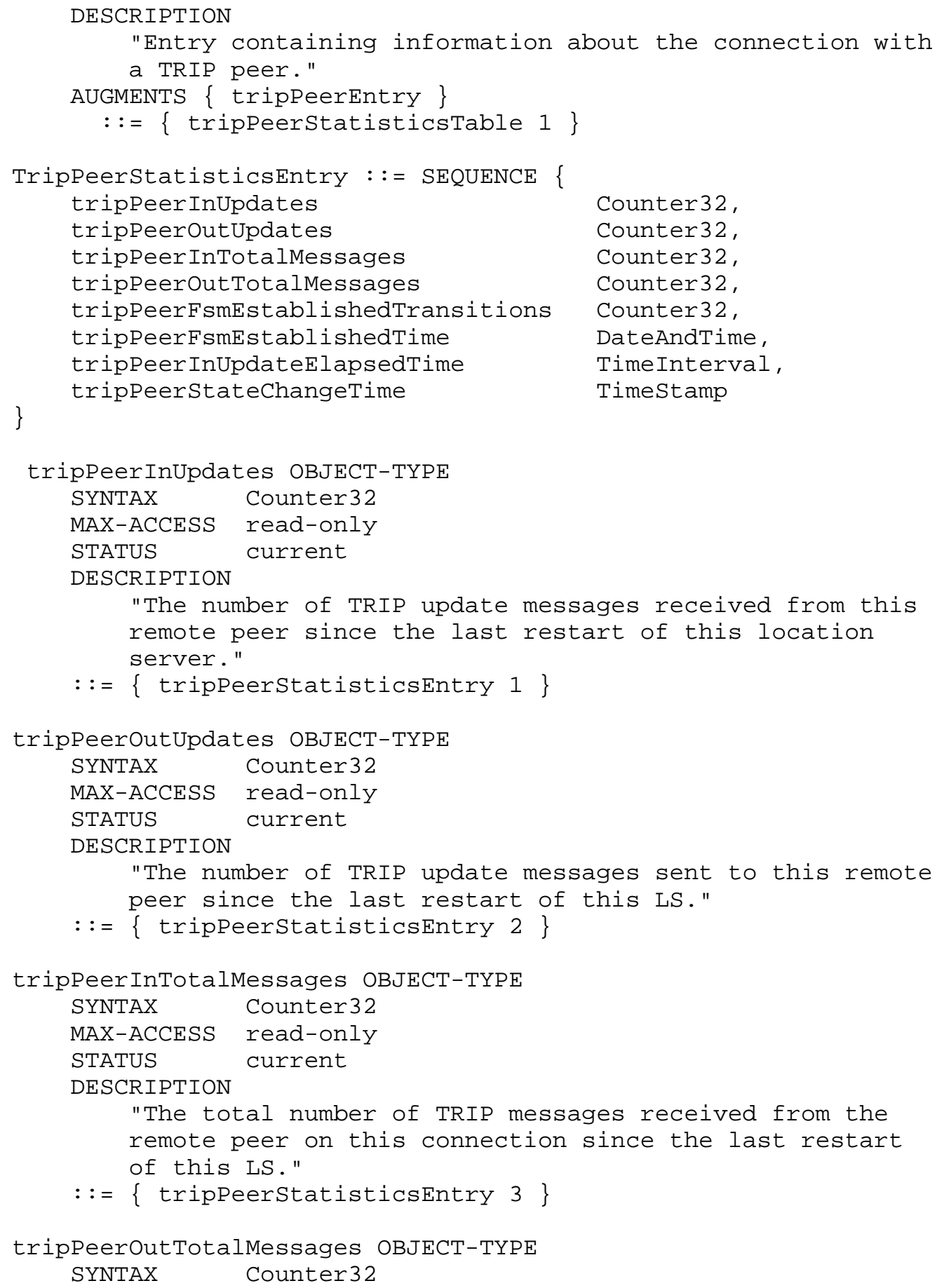




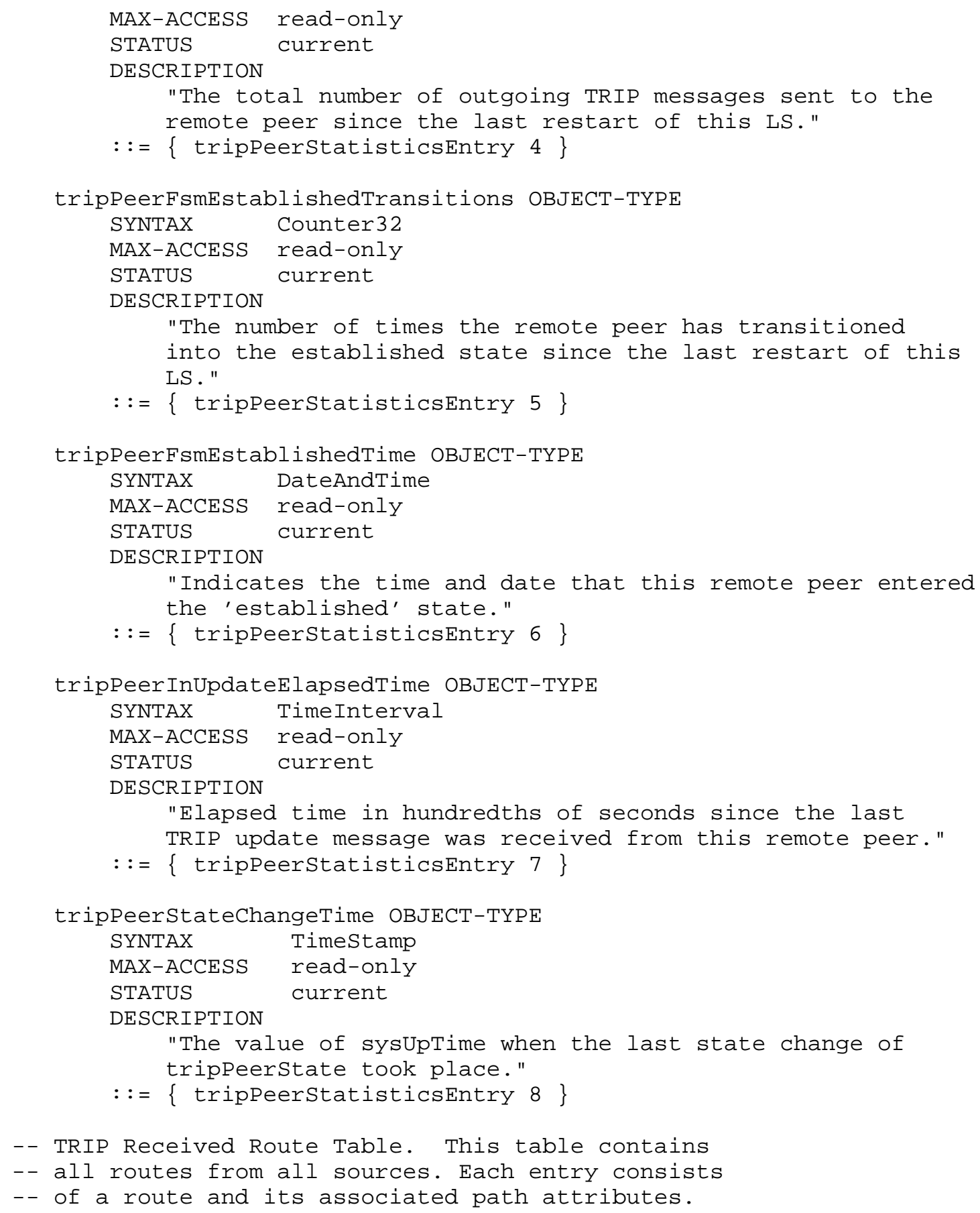




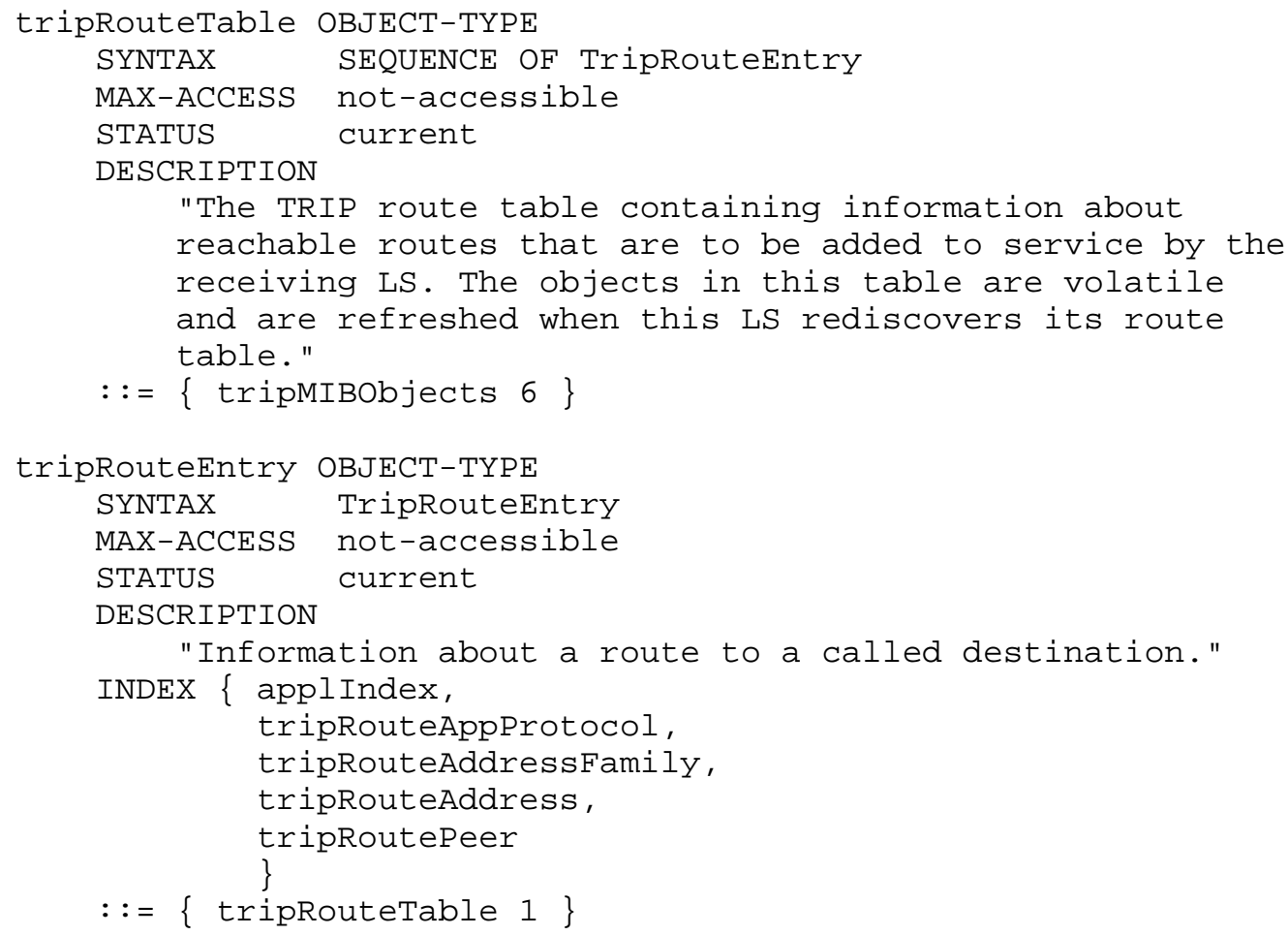




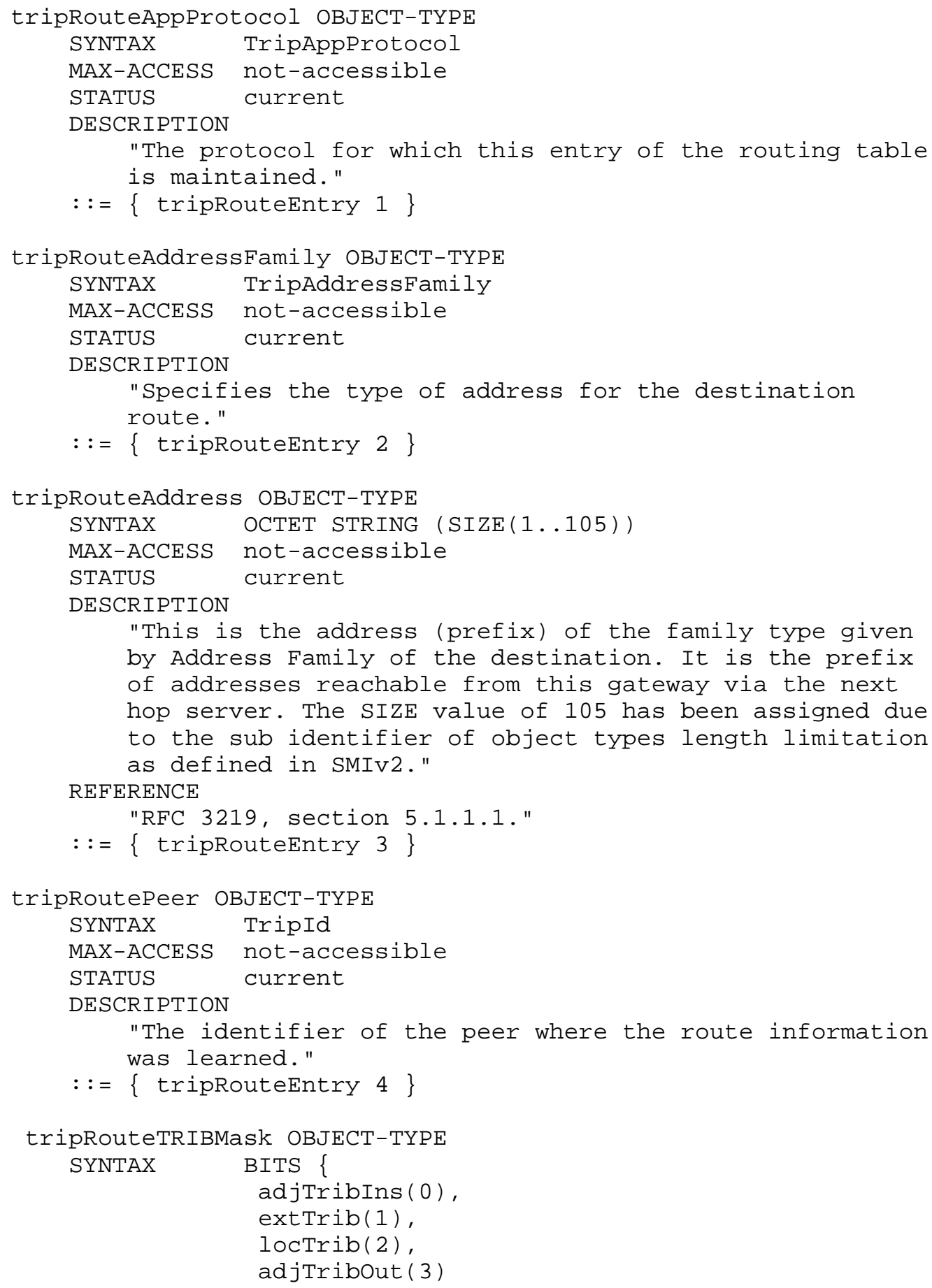




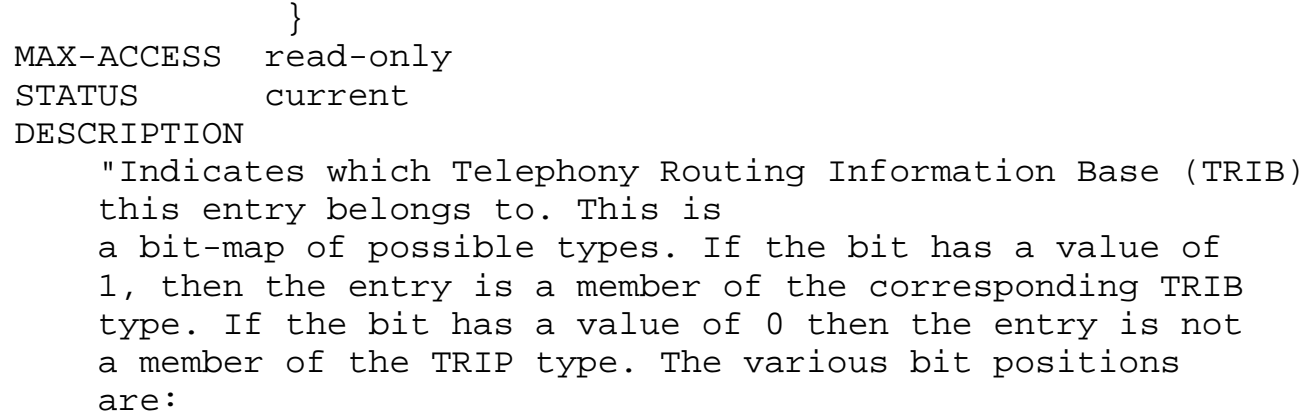




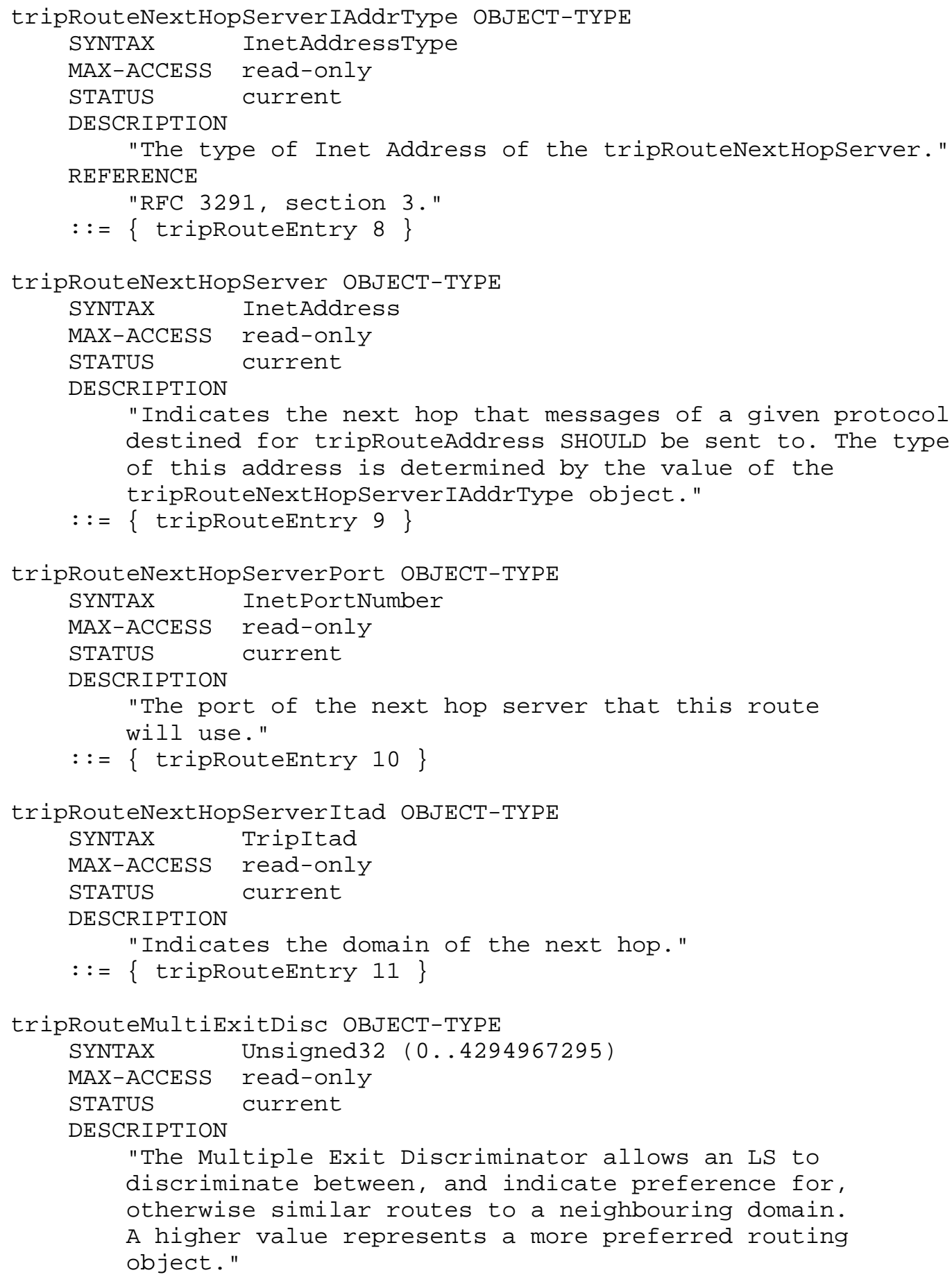




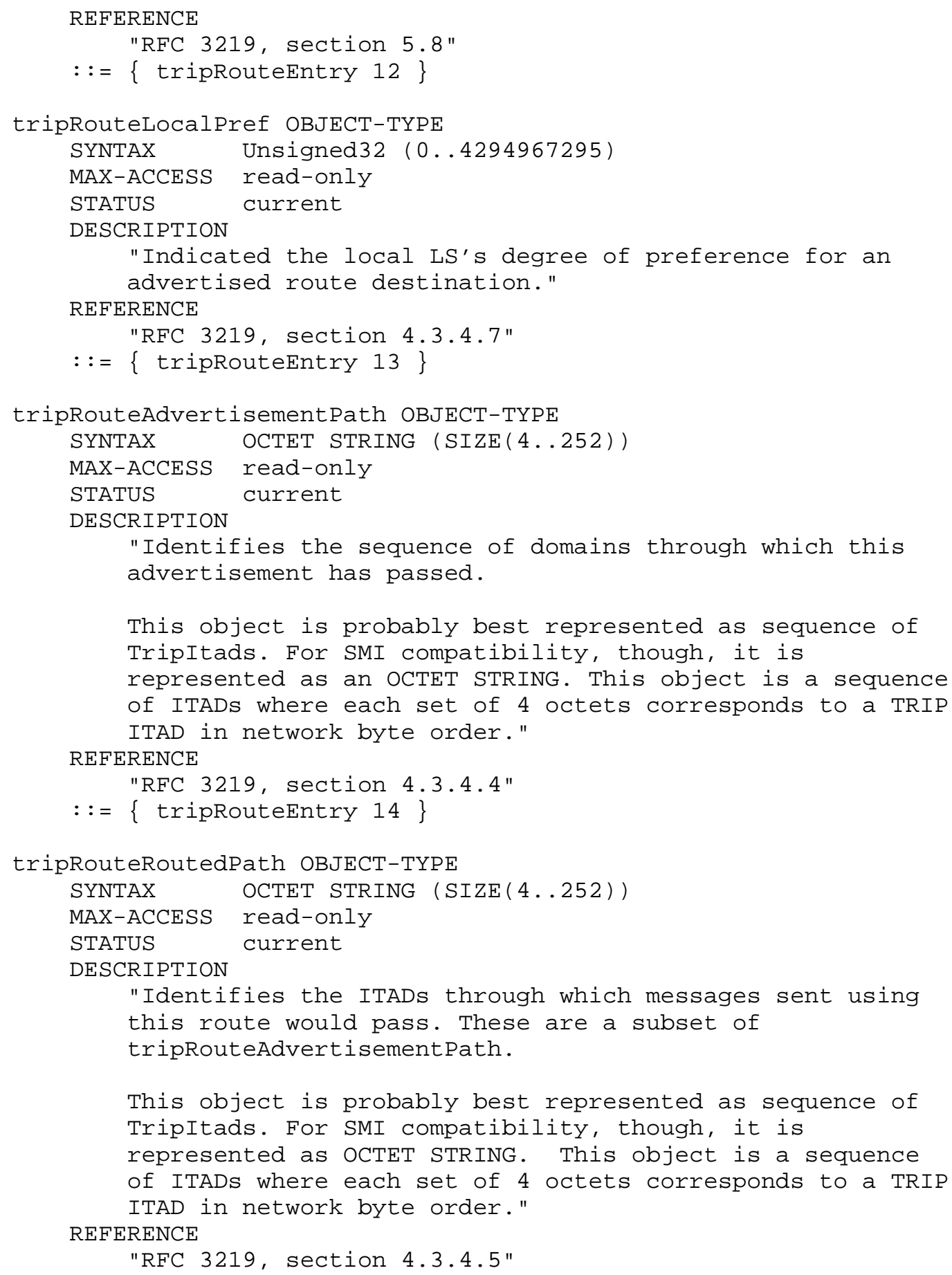


$::=\{$ tripRouteEntry 15$\}$

tripRouteAtomicAggregate OBJECT-TYPE

SYNTAX TruthValue

MAX-ACCESS read-only

STATUS current

DESCRIPTION

"Indicates that a route MAY traverse domains not listed in tripRouteRoutedPath. If an LS selects the less specific route from a set of overlapping routes, then this value returns TRUE." REFERENCE

"RFC 3219, section 4.3.4.6"

$::=\{$ tripRouteEntry 16$\}$

tripRouteUnknown OBJECT-TYPE

SYNTAX OCTET STRING (SIZE(0..255))

MAX-ACCESS read-only

STATUS current

DESCRIPTION

"This object contains one or more attributes that were not understood, and because they were transitive, were dropped during aggregation. They take the format of a triple <attribute type, attribute length, attribute value>, of variable length. If no attributes were dropped, this returns an OCTET STRING of size 0." REFERENCE

"RFC 3219, sections 4.3.1, 4.3.2.3"

$::=\{$ tripRouteEntry 17$\}$

tripRouteWithdrawn OBJECT-TYPE

SYNTAX TruthValue

MAX-ACCESS read-only

STATUS current

DESCRIPTION

"Indicates if this route is to be removed from service by the receiving LS."

$::=\{$ tripRouteEntry 18$\}$

tripRouteConverted OBJECT-TYPE

SYNTAX TruthValue

MAX-ACCESS read-only

STATUS current

DESCRIPTION

"Indicates if this route has been converted to a different application protocol than it had originally." $::=\{$ tripRouteEntry 19$\}$ 


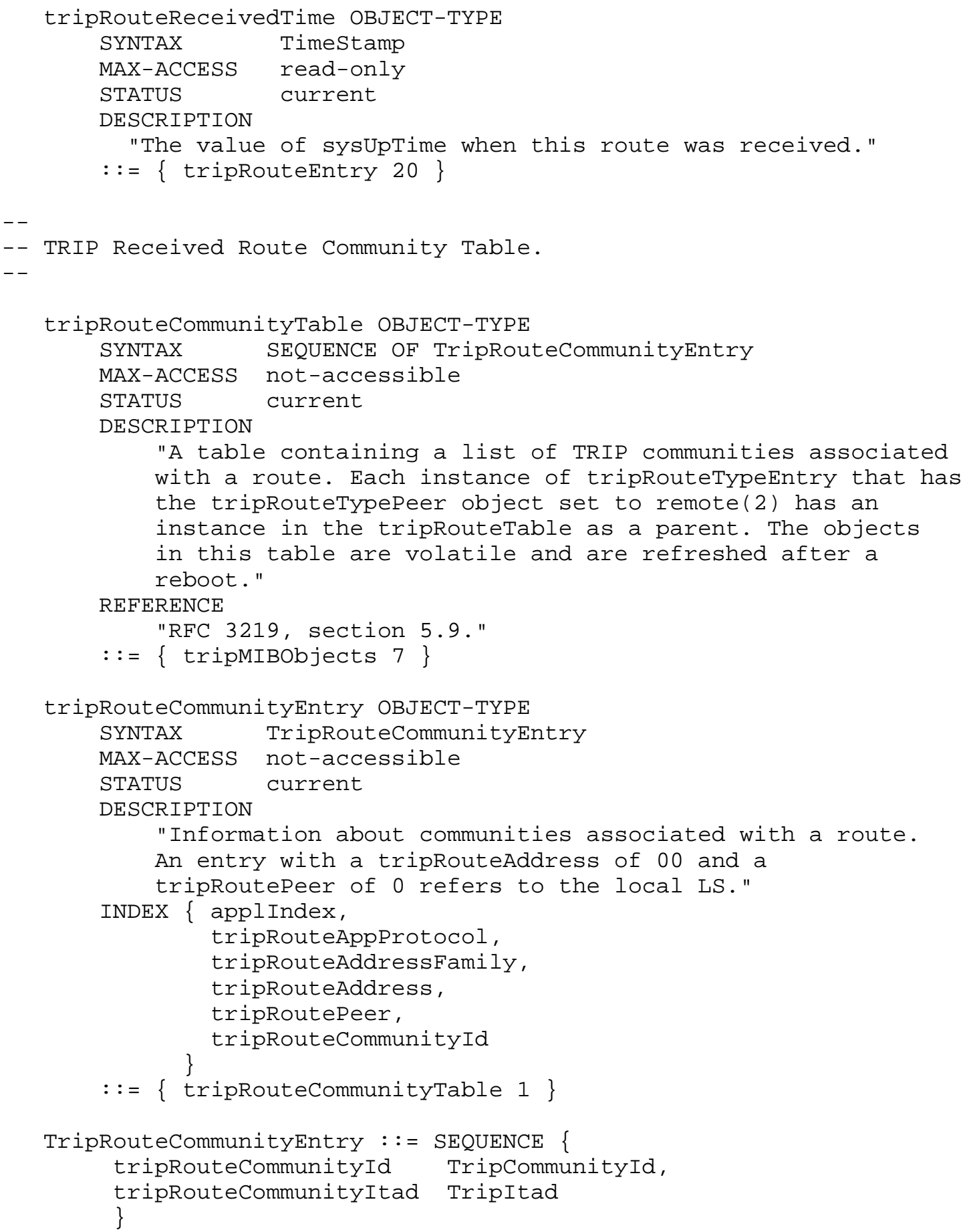




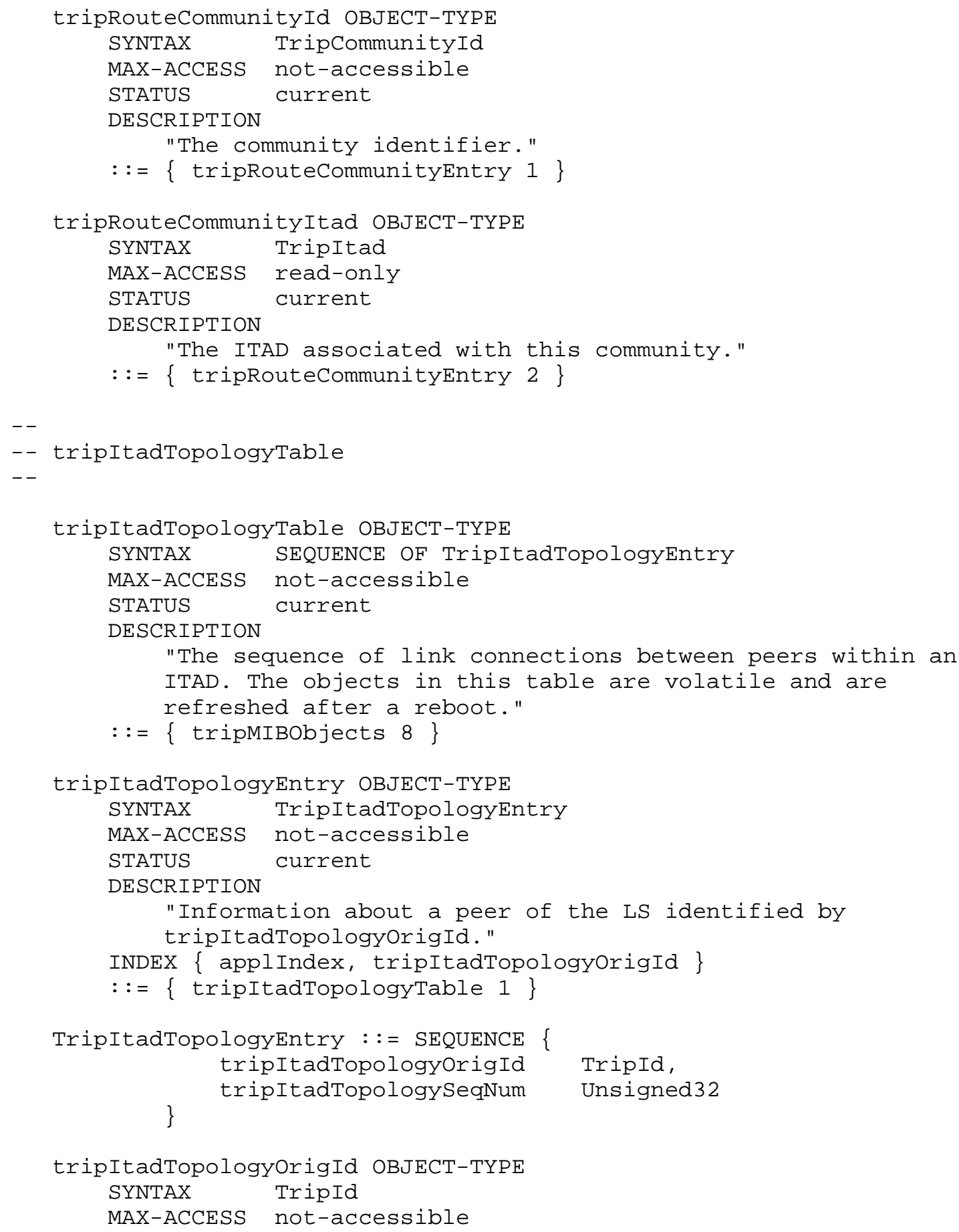




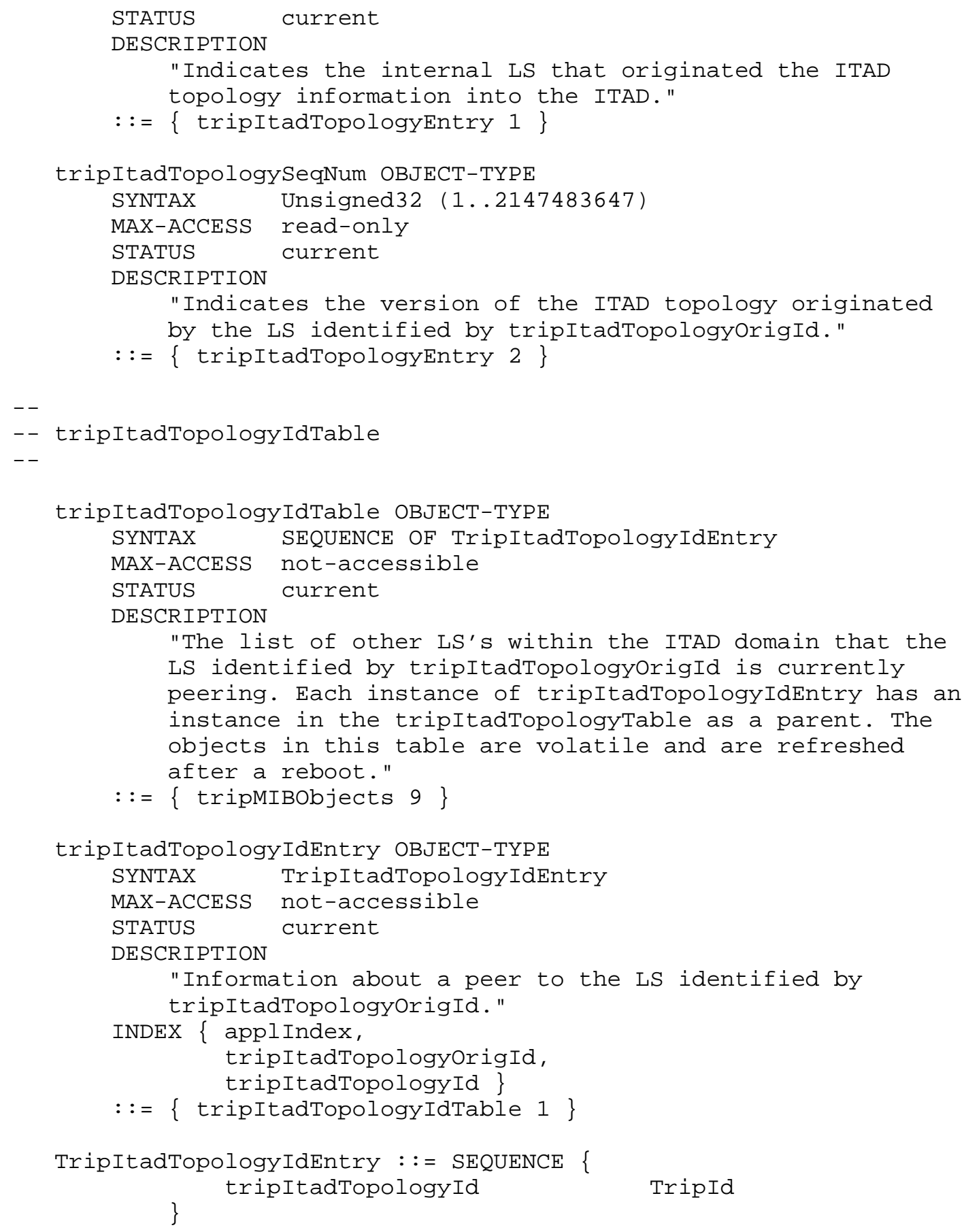




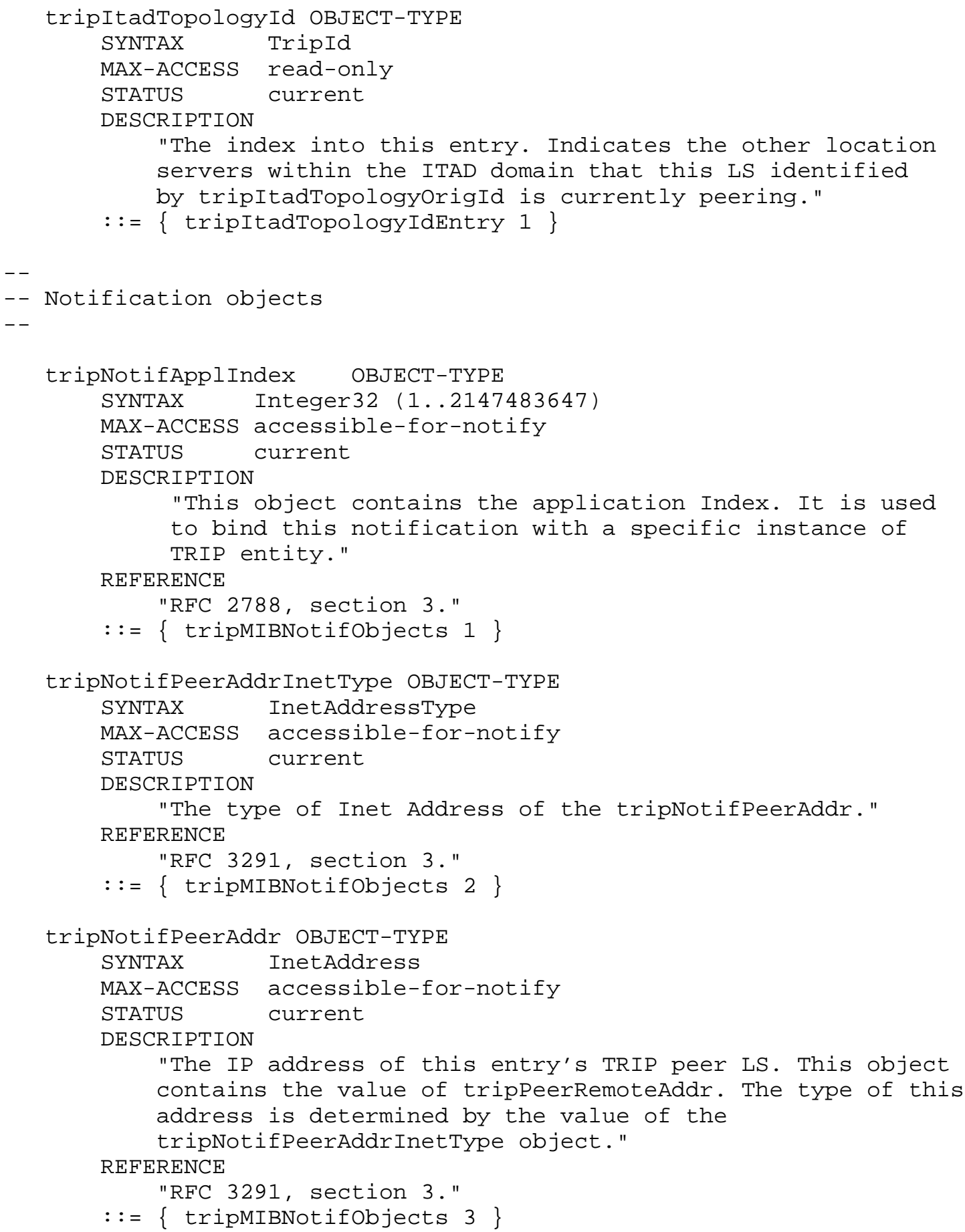




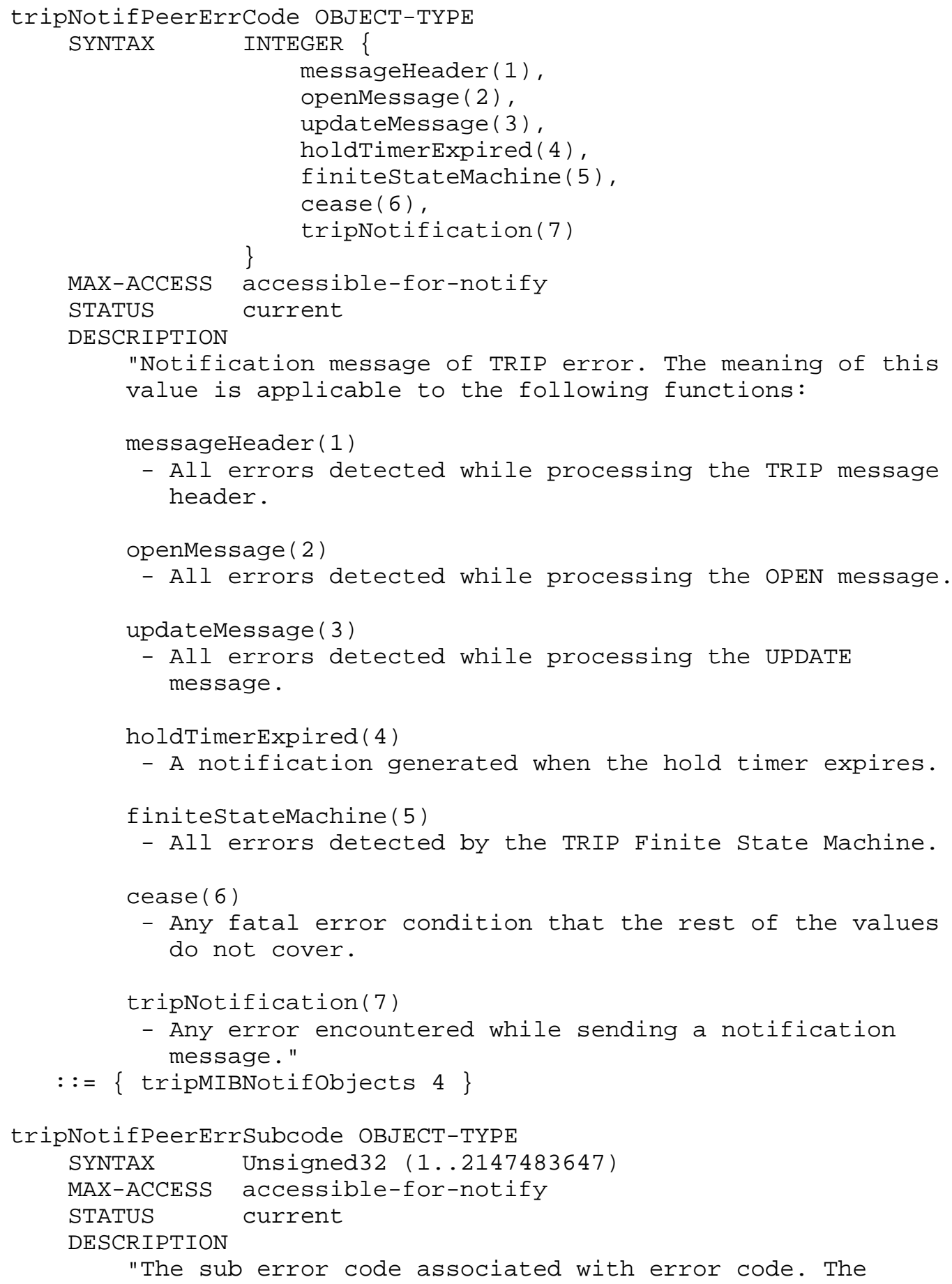




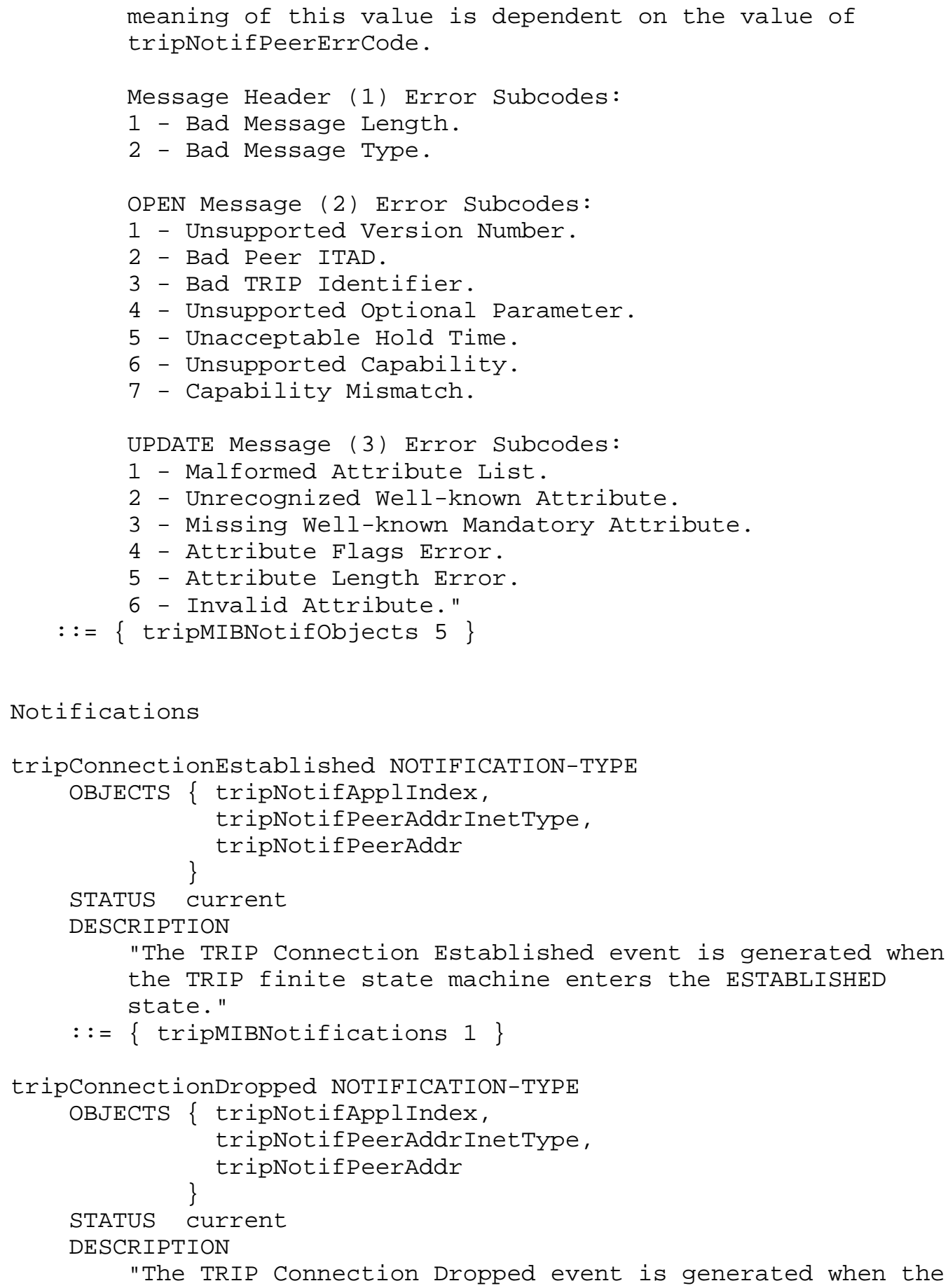


TRIP finite state machine leaves the ESTABLISHED state." $::=\{$ tripMIBNotifications 2$\}$

tripFSM NOTIFICATION-TYPE

OBJECTS \{ tripNotifApplIndex, tripNotifPeerAddrInet Type, tripNotifPeerAddr, tripNotifPeerErrCode, tripNotifPeerErrSubcode, \} trippeerstate

STATUS current

DESCRIPTION

"The trip FSM Event is generated when any error is detected by the TRIP Finite state Machine."

$::=\{$ tripMIBNotifications 3$\}$

tripopenMessageError NOTIFICATION-TYPE

OBJECTS \{ tripNotifApplIndex,

tripNotifPeerAddrInet Type, tripNotifPeerAddr, tripNotifPeerErrCode, tripNotifPeerErrSubcode, \} trippeerstate

STATUS current

DESCRIPTION

"Errors detected while processing the OPEN message." $::=\{$ tripMIBNotifications 4$\}$

tripUpdateMessageError NOTIFICATION-TYPE

OBJECTS \{ tripNotifApplIndex,

tripNotifPeerAddrInet Type, tripNotifPeerAddr, tripNotifPeerErrCode, tripNotifPeerErrSubcode, \} trippeerstate

STATUS current

DESCRIPTION

"Errors detected while processing the UPDATE message." $::=\{$ tripMIBNotifications 5$\}$

tripHoldTimerExpired NOTIFICATION-TYPE

OBJECTS \{ tripNotifApplIndex,

tripNotifPeerAddrInet Type,

tripNotifPeerAddr,

tripNotifPeerErrCode, 


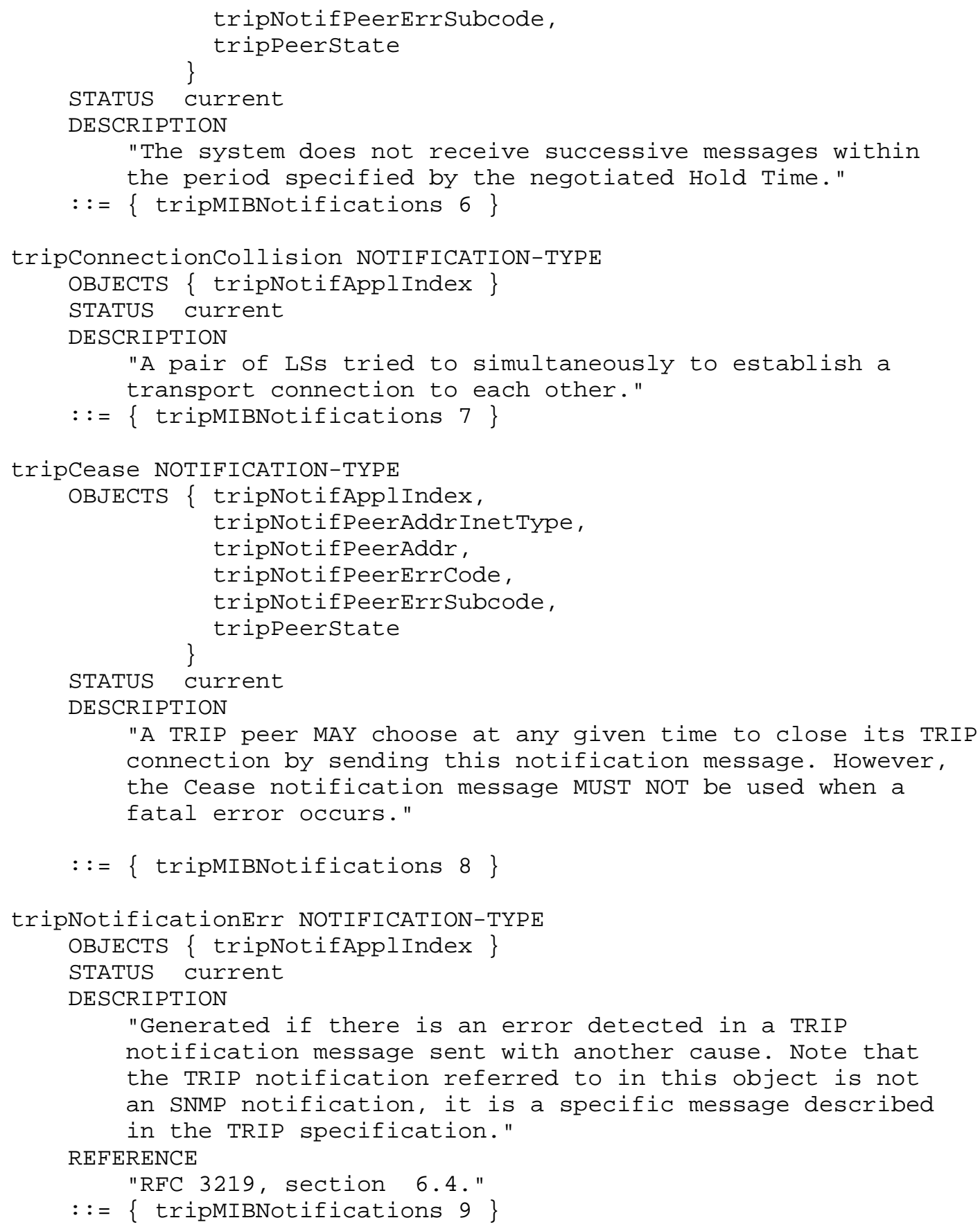


-- Compliance Statements

$--$

tripMIBFullCompliance MODULE-COMPLIANCE

STATUS current

DESCRIPTION

"The compliance statement for TRIP entities that

implement this MIB module in read-write mode, such

that it can be used for both monitoring and configuring

the TRIP entity.

There is one INDEX object that cannot be represented in

the form of OBJECT clauses in SMIv2, but for which there is a compliance requirement, expressed in OBJECT clause form in this description:

-- OBJECT tripRouteTypeAddrInetType

-- SYNTAX InetAddressType (ipv4(1), ipv6(2),

- DESCRIPTION

ipv4z(3), ipv6z(4))

-- This MIB requires support for global and

-- non-global ipv4 and ipv6 addresses.

$--$

- OBJECT tripRouteTypeAddr

$\left.\begin{array}{ll|l|l|l}-- & \text { SYNTAX InetAddress (SIZE (4 } & 8 & 16 & 20)\end{array}\right)$

-- DESCRIPTION

-- This MIB requires support for global and

-- non-global IPv4 and IPv6 addresses.

-

MODULE -- this module

MANDATORY-GROUPS \{ tripConfigGroup,

trippeerTableconfigGroup,

tripRouteGroup,

tripItadTopologyGroup,

tripPeerTablestatsGroup \}

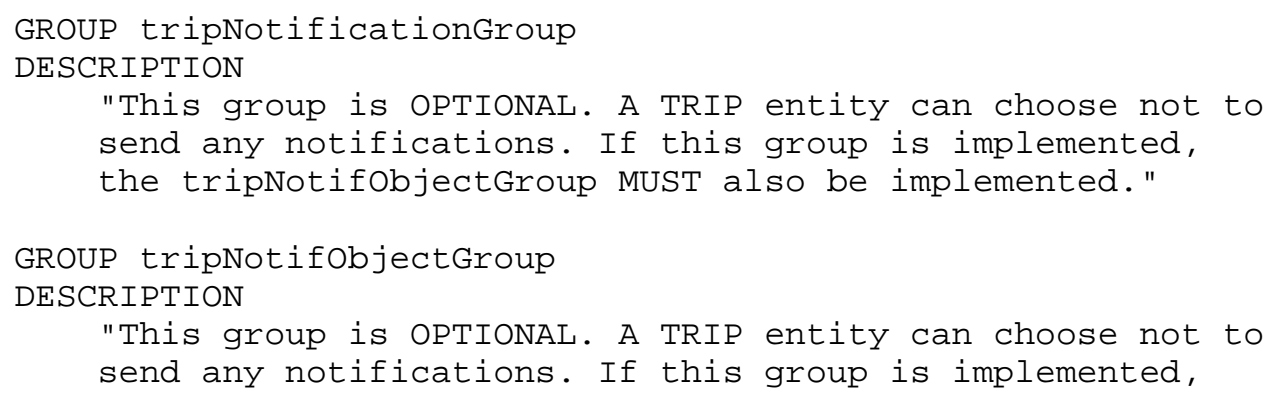


the tripNotificationGroup MUST also be implemented."

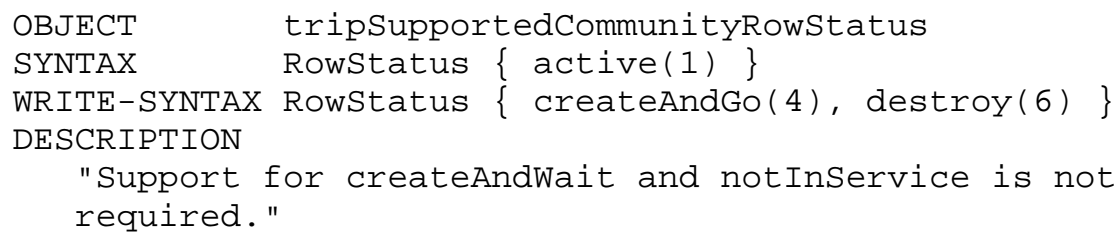




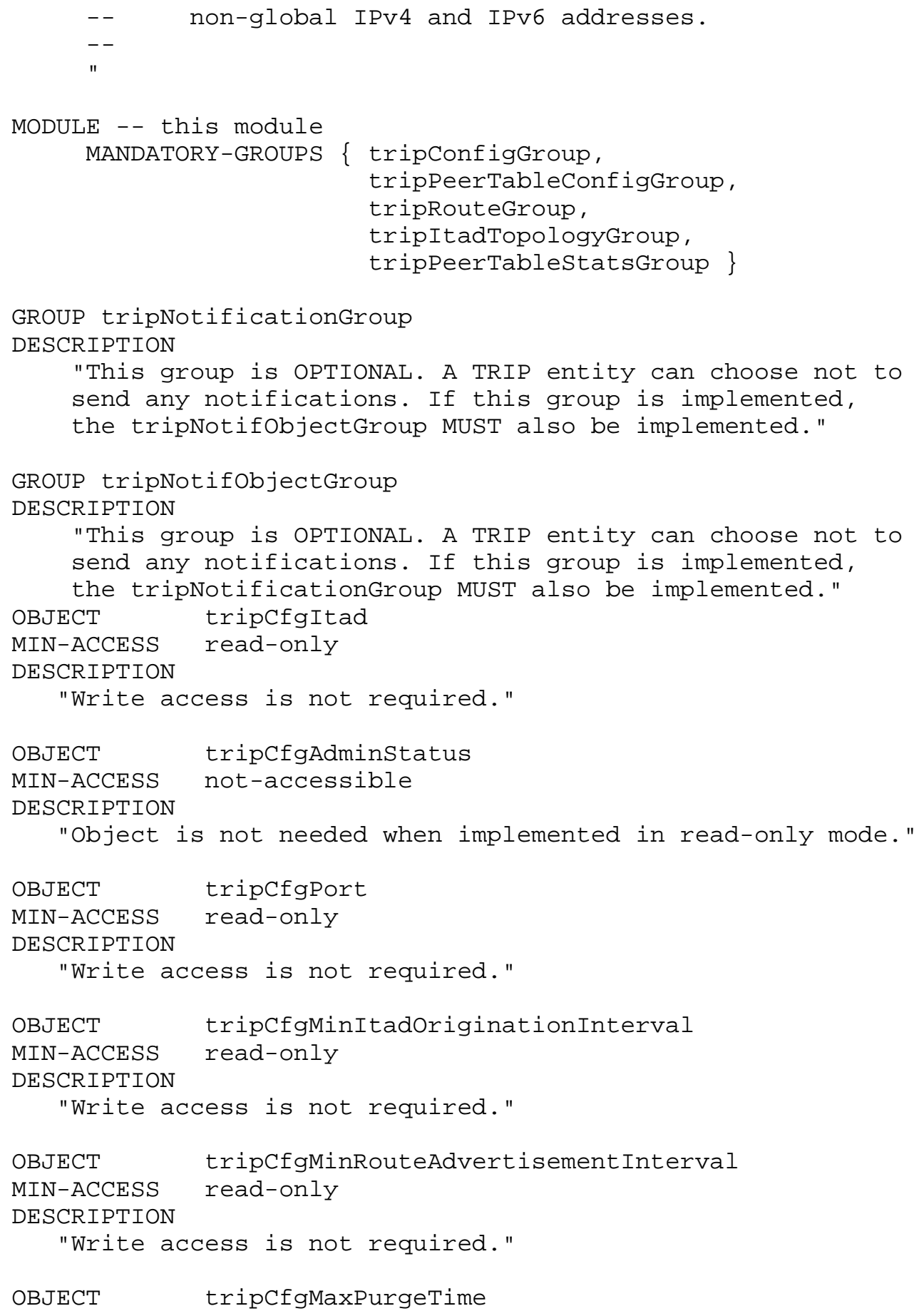




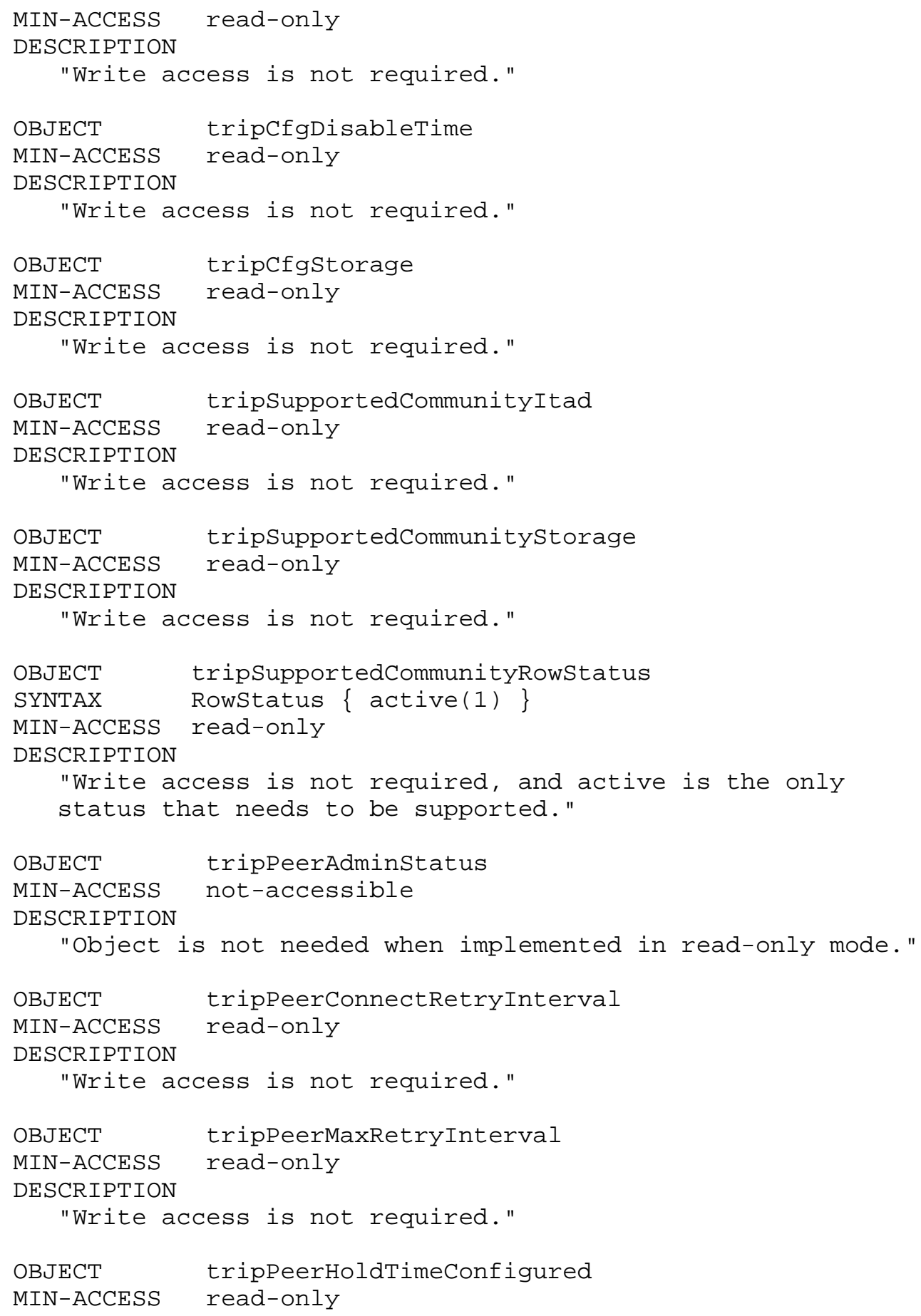




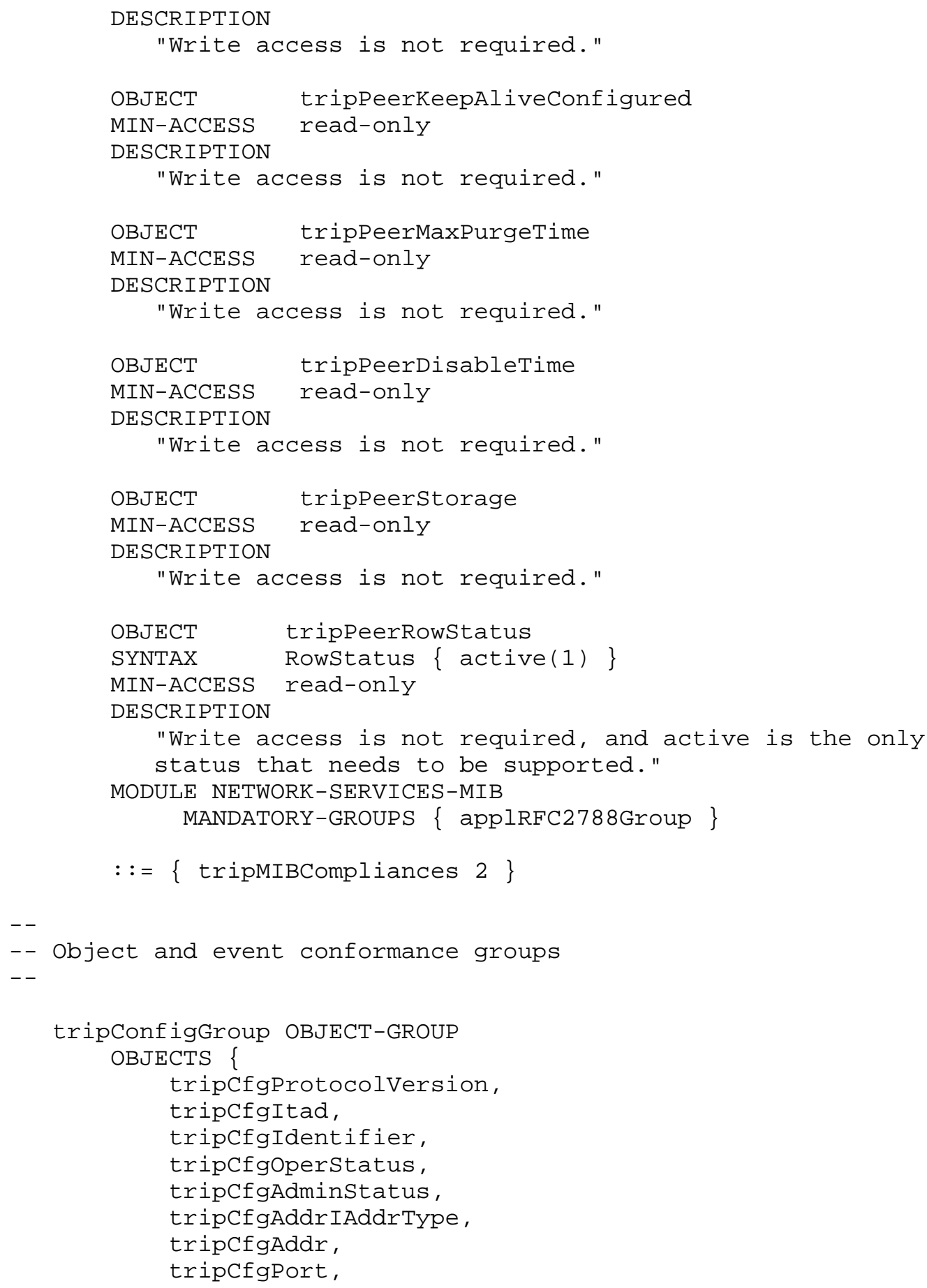




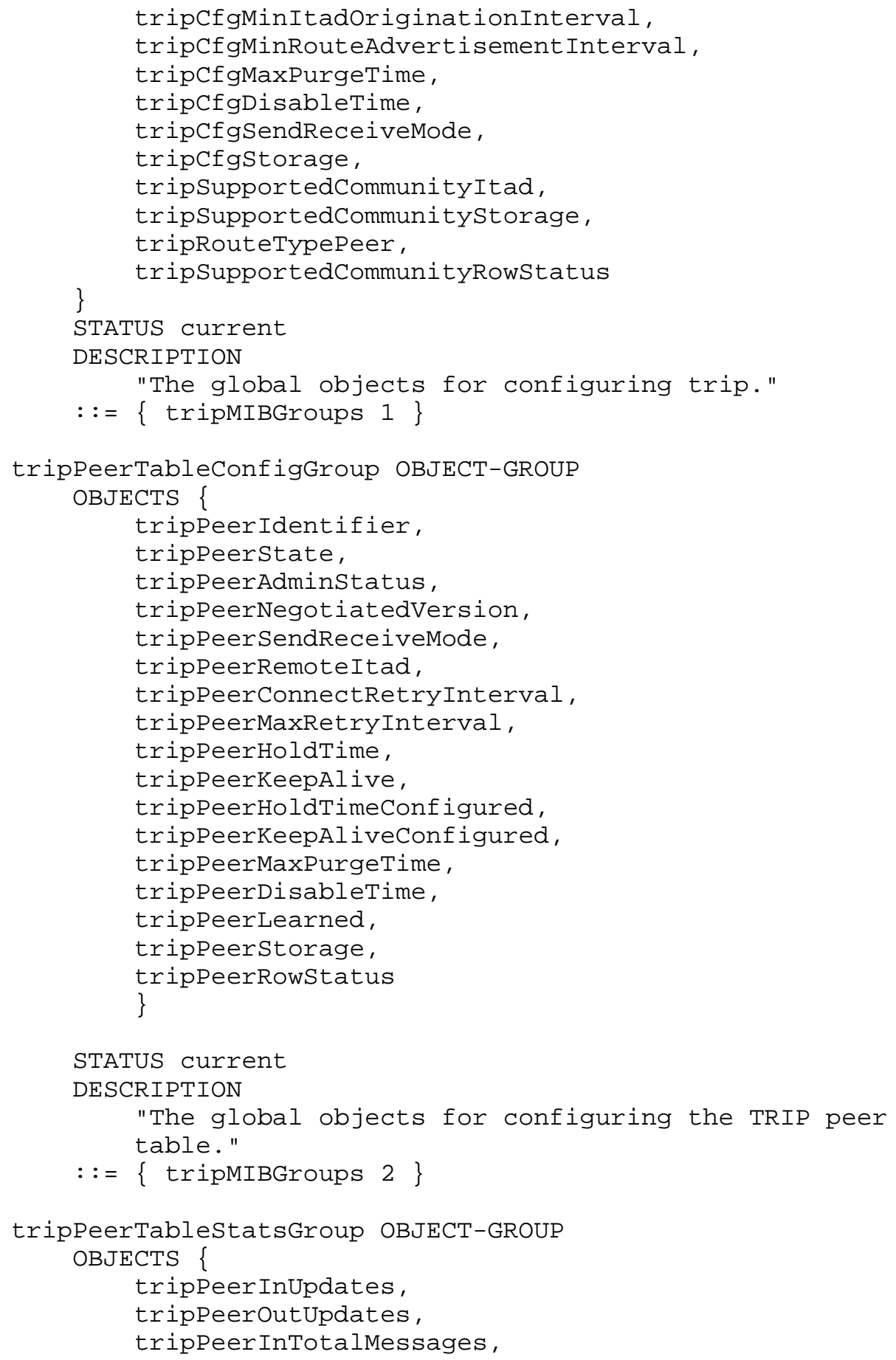




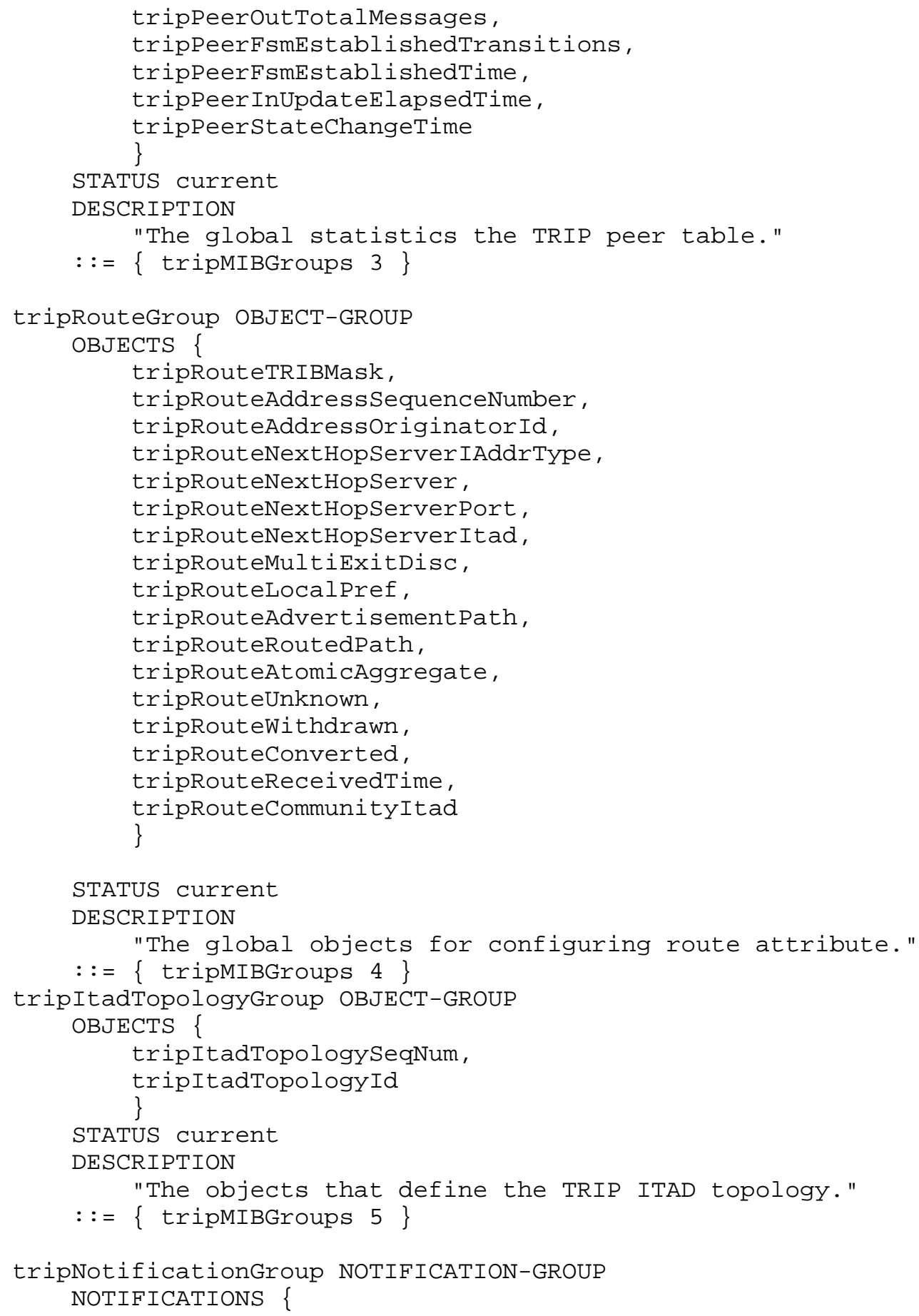




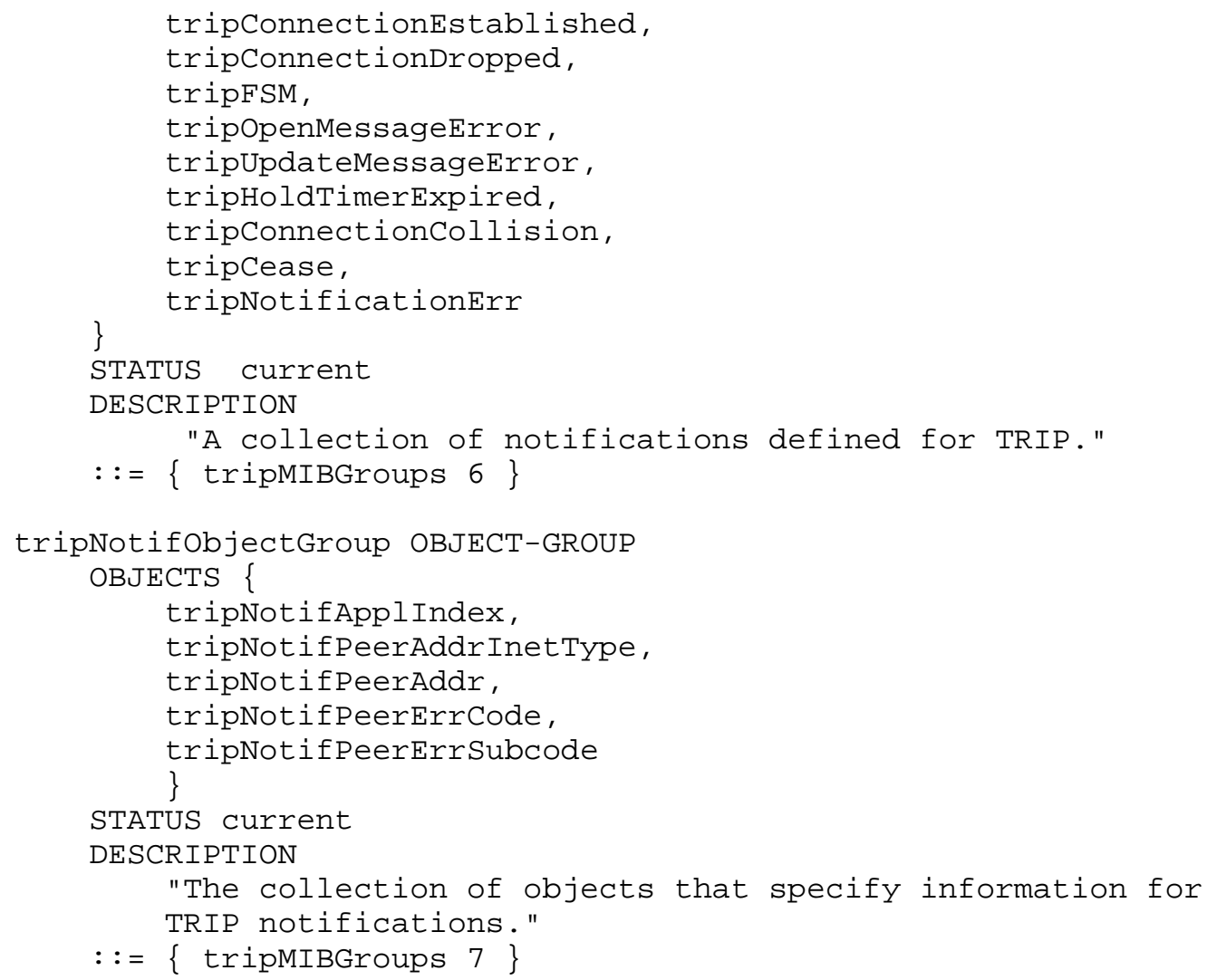

END

7. Security Considerations

The managed objects in this MIB module contain sensitive information since, collectively, they allow tracing and influencing of connections in TRIP devices and provide information of their connection characteristics. As such, improper manipulation of the objects represented by this MIB module MAY result in denial of service to a large number of available routes.

There are a number of management objects defined in this MIB module that have a MAX-ACCESS clause of read-write and/or read-create. Such objects MAY be considered sensitive or vulnerable in some network environments. The support for SET operations in a non-secure environment without proper protection can have a negative effect on network operations. These objects include: 


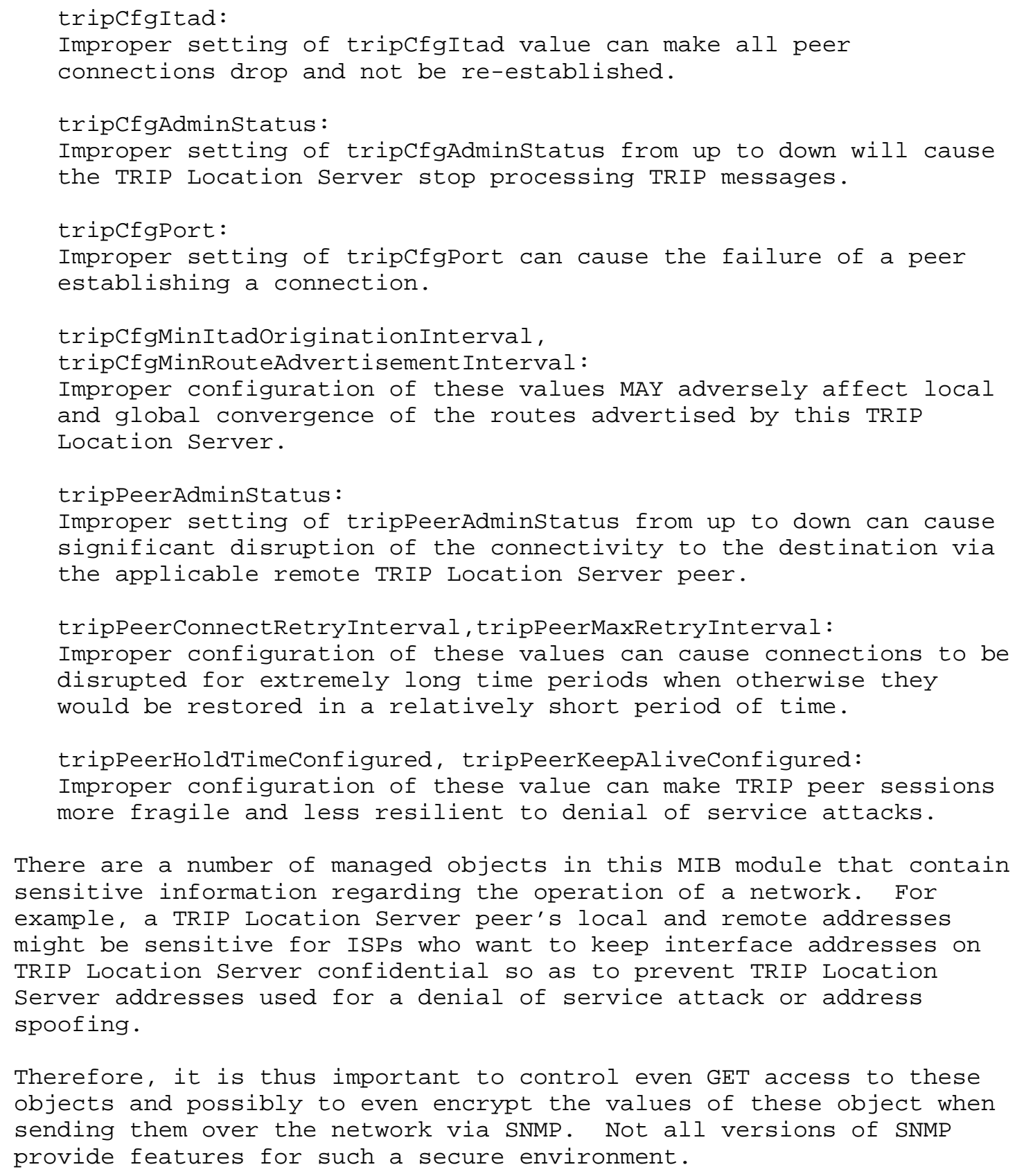


SNMPv1 by itself is not a secure environment. Even if the network itself is secure (for example by using IPSec), even then, there is no control as to who on the secure network is allowed to access and GET/SET (read/change/create/delete) the objects in this MIB module.

It is RECOMMENDED that the implementers consider the security features as provided by the SNMPv3 framework (see [RFC3410], section 8), including full support for the SNMPv3 cryptographic mechanisms (for authentication and privacy).

Further, deployment of SNMP versions prior to SNMPv3 is NOT RECOMMENDED. Instead, it is RECOMMENDED to deploy SNMPv3 and to enable cryptographic security. It is then a customer/operator responsibility to ensure that the SNMP entity giving access to an instance of this MIB module is properly configured to give access to the objects only to those principals (users) that have legitimate rights to indeed GET or SET (change/create/delete) them.

8. References

8.1. Normative References

[RFC2119] Bradner, S., "Key words for use in RFCs to Indicate Requirement Levels", BCP 14, RFC 2119, March 1997.

[RFC2579] McCloghrie, K., Perkins, D., and J. Schoenwaelder, "Textual Conventions for SMIv2", STD 58, RFC 2579, April 1999.

[RFC2580] McCloghrie, K., Perkins, D., and J. Schoenwaelder, "Conformance statements for SMIv2", STD 58, RFC 2580, April 1999.

[RFC2788] Freed, N. and S. Kille, "Network Services Monitoring MIB", RFC 2788, March 2000.

[RFC3219] Rosenberg, J., Salama, H., and M. Squire, "Telephony Routing over IP (TRIP)", RFC 3219, January 2002.

[RFC3291] Daniele, M., Haberman, B., Routhier, S., and J. Schoenwaelder, "Textual Conventions for Internet Network Addresses", RFC 3291, May 2002.

[RFC2578] McCloghrie, K., Perkins, D., and J. Schoenwaelder, "Structure of Management Information Version 2 (SMIv2)", STD 58, RFC 2578, April 1999. 


\subsection{Informative References}

[RFC1657] Willis, S., Burruss, J., and J. Chu, Ed., "Definitions of Managed Objects for the Fourth Version of the Border Gateway Protocol (BGP-4) using SMIv2", RFC 1657, July 1994.

[RFC1771] Rekhter, Y. and T. Li, "Border Gateway Protocol 4 (BGP-4)", RFC 1771, March 1995.

[RFC3410] Case, J., Mundy, R., Partain, D., and B. Stewart, "Introduction and Applicability Statements for InternetStandard Management Framework", RFC 3410, December 2002.

9. Acknowledgments

The authors wish to thank Bert Wijnen, Dan Romascanu, and Jonathan Rosenberg for their insightful comments and suggestions.

Thanks to Kevin Lingle for his invaluable comments, help with MIB things and great ideas. 
10. Authors' Addresses

David Zinman

Editor

265 Ridley Blvd

Toronto ON M5M 4N8

Canada

Phone: +1 4164334298

EMail: dzinman@rogers.com

David Walker

Sedna Wireless Inc.

495 March Road, Suite 500

Ottawa, ON K2K $3 \mathrm{G} 1$

Canada

Phone: +1 $613878 \quad 8142$

EMail: david.walker@sedna-wireless.com

Jianping Jiang

Syndesis Limited

30 Fulton Way

Richmond Hill, ON L4B 1J5

Canada

Phone: +1 905 886-7818 x2515

EMail: jjiangesyndesis.com 
11. Full Copyright statement

Copyright (C) The Internet Society (2004).

This document is subject to the rights, licenses and restrictions contained in BCP 78, and except as set forth therein, the authors retain all their rights.

This document and the information contained herein are provided on an "AS IS" basis and THE CONTRIBUTOR, THE ORGANIZATION HE/S HE REPRESENTS OR IS SPONSORED BY (IF ANY), THE INTERNET SOCIETY AND THE INTERNET ENGINEERING TASK FORCE DISCLAIM ALL WARRANTIES, EXPRESS OR IMPLIED, INCLUDING BUT NOT LIMITED TO ANY WARRANTY THAT THE USE OF THE INFORMATION HEREIN WILL NOT INFRINGE ANY RIGHTS OR ANY IMPLIED WARRANTIES OF MERCHANTABILITY OR FITNESS FOR A PARTICULAR PURPOSE.

Intellectual Property

The IETF takes no position regarding the validity or scope of any Intellectual Property Rights or other rights that might be claimed to pertain to the implementation or use of the technology described in this document or the extent to which any license under such rights might or might not be available; nor does it represent that it has made any independent effort to identify any such rights. Information on the IETF's procedures with respect to rights in IETF Documents can be found in $\mathrm{BCP} 78$ and $\mathrm{BCP} 79$.

Copies of IPR disclosures made to the IETF Secretariat and any assurances of licenses to be made available, or the result of an attempt made to obtain a general license or permission for the use of such proprietary rights by implementers or users of this specification can be obtained from the IETF on-line IPR repository at http://www.ietf.org/ipr.

The IETF invites any interested party to bring to its attention any copyrights, patents or patent applications, or other proprietary rights that may cover technology that may be required to implement this standard. Please address the information to the IETF at ietfipreietf.org.

Acknowledgement

Funding for the RFC Editor function is currently provided by the Internet Society. 